\title{
A BSP algorithm for on-the-fly checking CTL* formulas on security protocols
}

\author{
Frédéric Gava · Franck Pommereau . \\ Michaël Guedj
}

Received: date / Accepted: date

\begin{abstract}
This paper presents a distributed (Bulk-Synchronous Parallel or BSP) algorithm to compute on-the-fly whether a structured model of a security protocol satisfies or not a $\mathrm{CTL}^{*}$ formula. Using the structured nature of the security protocols allows us to design a simple method to distribute the state-space under consideration in a need-driven fashion. Based on this distribution of the states, the algorithm for logical checking of a LTL formula can be simplified and optimised allowing, with few tricky modifications, the design of an efficient algorithm for $\mathrm{CTL}^{*}$ checking. Some prototype implementations have been developed, allowing to run benchmarks to investigate the parallel behaviour of our algorithms.
\end{abstract}

Keywords BSP $\cdot \mathrm{LTL} \cdot \mathrm{CTL}^{*} \cdot$ Security Protocols $\cdot$ State-space $\cdot$ Model-checking

\section{Introduction}

In a world strongly dependent on distributed data communication, the design of secure infrastructures is a crucial task. At the core of computer security-sensitive applications are security protocols, i.e., sequences of message exchanges aiming at distributing data in a cryptographic way to the intended users and providing security guarantees such as confidentiality of data, authentication of participants, etc. This leads to search for a way to verify whether a protocol is secure or not [16].

But designing and verifying secure protocols is a challenging problem. In spite of their apparent simplicity, they are notoriously error-prone. Attacks exploit weaknesses in the protocol that are due to the complex and unexpected interleaving of different protocol sessions generated by a malicious intruder which resides in the network. A

Frédéric Gava and Michaël Guedj

LACL, University of Paris-East, Créteil, France

E-mail: frederic.gava@univ-paris-est.fr

Franck Pommereau

IBISC, University of Évry, France

E-mail: franck.pommereau@ibisc.univ-evry.fr 
famous example is the "man-the-middle" attack on the Needham-Schroeder public key protocol. The intruder is assumed to have a complete control on the network and to be powerful enough to perform potentially dangerous actions such as intercepting messages flowing over the network, or replacing them by new ones using the knowledge he has previously gained [20].

\subsection{Model-checking security protocols}

Unfortunately, the question of whether a protocol achieves its security requirements or not is, in the general case, undecidable or NP-complet in the case of a bounded number of agents [6]. Even if security protocols should theoretically be checked under an unbounded number of concurrent protocol executions, violating their security requirements often exploits only a small number of sessions (that is, an execution of an instance of the protocol) and agents. For these reasons, it is in many cases of interest sufficient to consider a finite number of sessions (in which each agent performs a fixed number of steps) in order to find flaws (which is distinct from proving the protocol). Formal methods offer a promising approach for automated security analysis of protocols: the intuitive notions are translated into formal specifications, which is essential for a careful design and analysis. The development of formal techniques that can check various security properties is an important tool to meet this challenge [16]. Enumerative (explicit) model-checking is well-adapted to find flaws in this kind of asynchronous, non-deterministic systems $[6,2]$. In particular, when an execution of the protocol is discovered to violate a security property, it can be presented as a trace of the protocol execution, that is, an explicit attack scenario.

By focusing on the verification of a bounded number of sessions, model-checking a protocol can be done by simply enumerating and exploring all traces of the execution of the protocol and looking for a violation of some of the requirements. Verification through model-checking consists in defining a formal model of the system to be analysed and then using automated tools to check whether the expected properties (generally expressed in a temporal logic) are met or not on the state-space of the model. To do so, all the different configurations of the execution of the agents evolving in the protocol need to be computed [6].

In this paper, we consider the problem of checking in a distributed way formulas expressed in the temporal logic $\mathrm{CTL}^{*}$ over labelled transition systems (LTS) that model security protocols. Checking a logical formula over a protocol is not new [6, 1] and has the advantage over dedicated tools for protocols (such as PROVERIF [9] or SCYTHER [18] to cite the most known) to be easily extensible to non standard behaviour of honest principals (e.g., contract-signing protocols in which participants are required to make progress toward an agreement) or to check some security goals that cannot be expressed as reachability properties, e.g., fair exchange [6].

\subsection{Distributed model-checking: problematic and contribution}

But the greatest problem with explicit model checking in general (and for security protocols in particular) is the so-called state explosion: the fact that the number of 
states typically grows exponentially with the number of agents and sessions. This is especially true when complex data-structures are used in the model such as the knowledge of an intruder in a security protocol. Checking a $\mathrm{CTL}^{*}$ formula over a security protocol may thus be expensive both in terms of memory and execution time.

Because explicit model-checking can cause memory crashing on single or multiple processor systems, it has led to consider exploiting the larger memory space available in distributed systems [24], which also gives the opportunity to reduce the overall execution time. Parallelizing the state-space construction on several machines is thus done in order to benefit from each machine's complete storage and computing resources. One of the main technical issues is to partition the state space, i.e. each subset of states is "owned" by a single machine.

To have efficient parallel algorithms for this state-space construction, it is common to have the following requirements. First, how states are partitioned across the processors must be computed quickly. Second, the successor function (of a state) must be defined so that successors states are likely mapped to the same processor as its predecessor; otherwise the computation will be overwhelmed by inter-processor communications (the so-called cross transitions) which obviously implies a drop of the computation locality and thus of the performances. Third, balancing the workload is obviously needed [33] in order to fully profit from available computational power and to achieve the expected speedup. In the case of state-space construction, the problem is hampered by the fact that future size and structure of the undiscovered portion of the state-space are unknown and cannot be predicted in general. Moreover, during the state-space construction, all the explored states may need to be kept in memory in order to avoid multiple exploration of a same state. This can lead to fill the main memories and induce swapping which is known to significantly slow machines.

Furthermore, one may identify two basic approaches to model-checking. The first one uses a global analysis to determine if a system satisfies or not a formula; the entire state-space of the system is constructed and subjected latter to analysis. However, these algorithms may be used to perform unnecessary work because in many cases (especially when a system does not satisfy a specification), only a subset of the system states needs to be analysed in order to determine whether the system satisfies a formula or not. It is thus rarely necessary to compute the entire state-space before finding a path that invalidates the logic formula (a flaw in a protocol). On the other hand, on-the-fly (or local) approaches to model-checking attempt to take advantage of this observation by constructing the state-space in a demand-driven fashion: onthe-fly algorithms are designed to build the state-space and check the formula at the same time which is thus generally more efficient.

By exploiting the well-structured nature of security protocols, we propose a solution to simplify the writing of an efficient on-the-fly model-checking distributed algorithm for finite scenarios. The structure of the protocols is exploited to partition the state-space, to reduce cross transitions while increasing computation locality, to keep only a sub-part of the state-space in the main memories (to avoid swapping on external/disk memories) and to load balance the computations. At the same time, the BSP model of computation [8] (defined later in this paper) allows us to simplify the detection of the algorithm termination and to further load-balance the computations. 
Our work is based on the sequential algorithm of [7] which mainly combines the construction a proof-structure (a graph) together with a Tarjan's depth-first-search based SCC (Strongly Connected Components) algorithm for detecting on-the-fly a reachable accepting cycle in the underlying graph.

\subsection{Outline}

First, we briefly review in Section 2 the context of our work that is the BSP model, models of security protocols and their state-space representation as LTS, as well as the formal definition of two temporal logics LTL and CTL* together with their verification.

Section 3 is dedicated to the description of our new state-space algorithm constructed in a step-wise manner from a sequential one. Section 4 is dedicated to the design of a BSP algorithm for verification of a LTL formula on a security protocol and Section 5 is the generalisation of the above algorithm for CTL* . For all the algorithms, we briefly describe a prototype implementation and apply it to some typical protocol sessions, giving benchmarks to demonstrate the benefits of our approach.

Finally, related works are discussed in Section 6 while a conclusion and future works are presented in Section 7.

\section{Context and general definitions}

\subsection{The BSP model of parallel execution}

A BSP computer is seen as a set of uniform processor-memory pairs connected through a communication network allowing the inter-processor delivery of messages [8]. Clusters of PCs, multi-core, etc., can be considered as BSP computers.

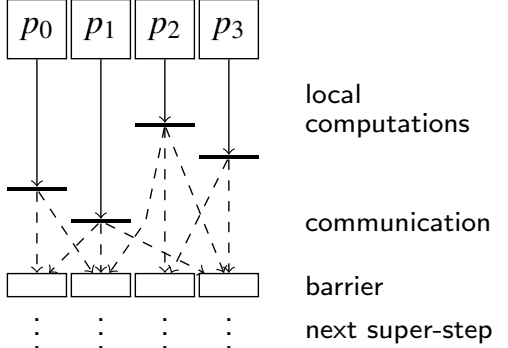

Fig. 1 A BSP super-step.

A BSP program is logically executed as a sequence of super-steps (see Fig. 1), each of which is divided into three successive disjoint phases: (1) Each processor only uses its local data to perform sequential computations and to request data transfers to other nodes;

(2) The network delivers the requested data; (3) A global synchronisation barrier occurs, making the transferred data available for the next super-step. The execution time (cost) of a super-step is the sum of the maximum of the local processing, the data delivery and the barrier times. The cost of a program is the total sum of the cost of its super-steps.

The BSP model considers communication actions en masse. This is less flexible than asynchronous messages, but easier to debug since there are many simultaneous communication actions in a classical parallel program, and their interactions are usually complex. Bulk sending also provides better performances since it is faster to send a block of data rather than individual data because of less network latency. 
This structured model of parallelism enforces a strict separation of communication and computation: during a super-step, no communication between the processors is allowed but only transfer requests, only at the synchronisation barrier information is actually exchanged. However, for better performances, a BSP library can send messages during the computation phase of a super-step, but this is hidden to programmers. On most cheaper distributed architectures, barriers often become more expensive when the number of processors increases. However, dedicated architectures make them much faster and they have also a number of attractions. In particular, this execution policy has the main advantage that it removes non-determinism and guarantees the absence of deadlocks since barriers do not create circular data dependencies. This is also merely the most visible aspects of a parallel model that shifts the responsibility for timing and synchronisation issues from the applications to the communications library. This can be used at runtime to dynamically make decisions, for instance choose whether to communicate in order to re-balance data, or to continue an unbalanced computation. BSP libraries are generally implemented using MPI or low level routines of the given specific architectures.

\subsection{Security protocols and their state-space}

\subsubsection{Brief overview of the security protocols}

Security protocols ${ }^{1}$ specify an exchange of cryptographic messages between principals, i.e., the agents (users, hosts, servers, etc.) participating in the protocol. Each instance of the protocol is called a session and an agent can participate to more than one session, sequentially or concurrently. A scenario is a particular choice of arrangement for different sessions involving a particular choice of agents. Messages are sent over open networks, such as the Internet, that are not secured. As a consequence, protocols should be designed to work fine even if messages may be eavesdropped or tampered with by an intruder - e.g., a dishonest or careless agent. Finally, each protocol is aimed to provide security guarantees such as authentication of principals or secrecy of some pieces of information (e.g., a key, a value that can crypt/decrypt a message or a nonce, a value that is new for each session) or non-repudiation and fairness for commercial protocol with a contract.

Agents perform "ping-pong" data exchanges and some well-known strategies that an intruder might employ are: man-in-the-middle, the intruder imposing itself in the communications between the sender and receiver; replay, the intruder monitors a run of the protocol and at some later time replays one or more of the messages; etc.

We assume the use of keys sufficiently long of the best-known cryptographic algorithms to prevent a brute force attack in a feasible time. This is the well-known perfect cryptography assumption. The idea is that an encrypted message can be decrypted only by using the appropriate decryption key, i.e., it is possible to retrieve $M$ from the message encrypted $\{M\}_{K}$ only by using $K^{-1}$ as decryption key and it is hopeless to compute $K^{-1}$ from $K$ or to guess one of these keys.

\footnotetext{
${ }^{1}$ More details on their modelling, semantics and attacks can be found in $[16,6]$.
} 

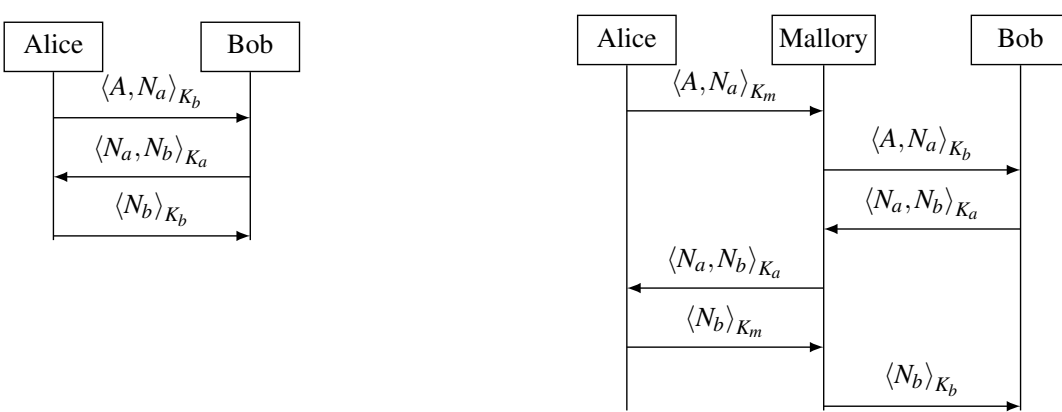

Fig. 2 The NS protocol (left) and the well-known "man-in-the-middle" attack (right).

Fig 2 (left) illustrates the standard Needham-Schroeder (NS) protocol which involves two agents Alice $(A)$ and Bob $(B)$ who want to mutually authenticate $-N_{a}$ and $N_{b}$ are nonces and $K_{a}, K_{b}$ are the public keys of respectively Alice and Bob. The idea of the protocol is that each agent sends a challenge to the other under the form of a nonce (unguessable by nature) encrypted with the receiver's public key who is this the only one able to decrypt the nonce and send it back. In the right of Fig 2, we show the well known flaw of the NS protocol when initiated with a malicious third party Mallory $(M)$; it involves two parallel sessions, with $M$ participating in both of them; Mallory authenticates as Alice with Bob. This is called a logical attack because both sessions of the protocol are correct but the overall security goals are not achieved.

In this paper, we thus consider that a Dolev/Yao attacker [20] resides on the network. An execution of such a model is thus a series of message exchanges as follows. (1) An agent sends a message on the network. (2) This message is captured by the attacker that tries to learn from it by recursively decomposing the message or decrypting it when the key to do so is known. Then, the attacker forges all possible messages from newly as well as previously learnt informations (i.e., attacker's knowledge). Finally, these messages (including the original one) are made available on the network. (3) The agents waiting for a message reception accept some of the messages forged by the attacker, according to the protocol rules. The Dolev/Yao threat model is a worstcase model in the sense that the network, over which the participants communicate, is thought as being totally controlled by an omnipotent intruder. Therefore, there is no need to assume the existence of multiple attackers, because they together do not have more abilities than the single Dolev/Yao intruder.

Model-checking attempts to find a reachable state or trace where a security property fails $-e . g$., secret term is learnt by the intruder or an incorrect authentication occurs. To ensure termination, these tools usually bound the maximum number of sessions. To model-check a security protocol, one must construct its state-space (all executions of the sessions) and check the property usually expressed in a temporal logic. We now formally define these steps.

\subsubsection{State-space construction}

The state-space construction problem is the problem of computing the explicit representation of a given model from the implicit one. In most cases, this space is con- 
structed by exploring all the states reachable through a successor function from an initial state. The state-space of a protocol thus includes all the executions of the sessions considering all the messages built by the intruder. The state-space (noted $S$ ) construction consists in constructing a LTS:

Definition 1 (Labelled Transition System, LTS) It is a tuple $(S, T, L)$ where $S$ is the set of states, $T \subseteq S \times S$ is the set of transitions, and $L$ is an arbitrary labelling on $S \cup T$.

Given a model implicitly defined by its initial state $s_{0}$ and its successor function succ, the corresponding explicit LTS $\left(s_{0}\right.$, succ $)$ is defined as the smallest LTS $(S, T, L)$ such that $s_{0} \in S$, and if $s \in S$, then for all $s^{\prime} \in \operatorname{succ}(s)$ we also have $s^{\prime} \in S$ and $\left(s, s^{\prime}\right) \in T$. The labelling may be arbitrarily chosen, for instance to define properties on states and transitions with respect to which model checking is performed. Now assuming a set $\mathscr{A}$ of atomic propositions, we have:

Definition 2 (Kripke structure) A Kripke structure is a LTS $(S, T, L)$ whose labelling is $L: S \rightarrow 2^{\mathscr{A}}$.

Mainly a Kripke structure is a LTS adjoining whose labelling function associates truth-values to the states.

Definition 3 (Path and related notions) Let $M \stackrel{\mathrm{df}}{=}(S, T, L)$ be a Kripke structure.

1. A path in $M$ is a maximal sequence of states $\left\langle s_{0}, s_{1}, \ldots\right\rangle$ such that for all $i \geq 0$, $\left(s_{i}, s_{i+1}\right) \in T$.

2. If $x=\left\langle s_{0}, s_{1}, \ldots\right\rangle$ is a path in $M$ then $x(i) \stackrel{\text { df }}{=} s_{i}$ and $x^{i} \stackrel{\text { df }}{=}\left\langle s_{i}, s_{i+1}, \ldots\right\rangle$.

3. If $s \in S$ then $\Pi_{M}(s)$ is the set of paths $x$ in $M$ such that $x(0)=s$.

\subsubsection{Properties of the state-spaces of security protocols}

In this paper, we model security protocols as LTS such that any state can be represented by a function from a set of locations to an arbitrary data domain. For instance, locations may correspond to local variables of agents, buffers, etc.

As a concrete formalism to model protocols, we have used an algebra of coloured Petri nets called ABCD [42] (not presented in this paper) allowing easy and structured modelling. This algebra is part of the SNAKES library [42] which is a general Petri net library that allows to model and execute PYTHON-coloured Petri nets: tokens are PYTHON objects and net inscriptions are PYTHON expressions. We refer to [26] for more details and examples of models of security protocols using ABCD.

However, our approach is largely independent of the chosen formalism and it is enough to assume that the following properties (P1) to (P4) hold.

(P1) locations can be partitioned into two sets $\mathscr{R}$ and $\mathscr{L}$, and LTS function succ can be partitioned into two functions $\operatorname{succ}_{\mathscr{R}}$ and $\operatorname{succ}_{\mathscr{L}}$ such that: for all state $s$ and all $s^{\prime} \in \operatorname{succ}(s)$, denoting by $\left.s\right|_{\mathscr{R}}$ the state $s$ restricted to the locations from $\mathscr{R}$, we have that $\left.s^{\prime}\right|_{\mathscr{R}}=\left.s\right|_{\mathscr{R}} \Longrightarrow s^{\prime} \in \operatorname{succ}_{\mathscr{L}}(s)$, and $\left.s^{\prime}\right|_{\mathscr{R}} \neq\left. s\right|_{\mathscr{R}} \Longrightarrow s^{\prime} \in \operatorname{succ}_{\mathscr{R}}(s)$. Intuitively, succ $\mathscr{R}$ corresponds to transitions upon which an agent (except the attacker) receives information and stores it, and $\mathscr{R}$ are the locations where these agents store the information they receive. 
(P2) there is an initial state $s_{0}$ and there exists a function slice from states to natural numbers (a measure) such that if $s^{\prime} \in \operatorname{succ}_{\mathscr{R}}(s)$ then there is no path from $s^{\prime}$ to any state $s^{\prime \prime}$ such that slice $(s) \leq \operatorname{slice}\left(s^{\prime \prime}\right)$ and slice $\left(s^{\prime}\right)=\operatorname{slice}(s)+1$. This is often called a sweep-line progression and corresponds to the fact that agents perform irreversible actions. In particular, a reception by an agent corresponds to an irreversible step in the sequence of messages forming the protocol.

(P3) there exists also a hash function $\mathrm{cpu}_{\mathscr{R}}$ from states to natural numbers such that for all state $s$ if $s^{\prime} \in \operatorname{succ}_{\mathscr{L}}(s)$ then $\operatorname{cpu}_{\mathscr{R}}(s)=\operatorname{cpu}_{\mathscr{R}}\left(s^{\prime}\right)$. This is to say that only information in $\mathscr{R}$ is taken into account to compute the hash of a state, and in particular, the knowledge of the intruder is not involved.

(P4) if $s_{1}, s_{2} \in \operatorname{succ}_{\mathscr{R}}(s)$ and $\operatorname{cpu}_{\mathscr{R}}\left(s_{1}\right) \neq \operatorname{cpu}_{\mathscr{R}}\left(s_{2}\right)$ then there is no possible path from $s_{1}$ to $s_{2}$ and vice versa. This means that the receptions of two distinct messages lead to distinct executions of the protocol, which is the case for instance when agents permanently store the information they have received (this always holds in practice).

On concrete models, it is generally easy to distinguish syntactically the transitions that correspond to a message reception in the protocol with information storage. Thus, is it easy to partition succ as above and, for virtually all models of classical protocols protocol, it is also easy to check that the above properties are satisfied. This is the case in particular for us using the $\mathrm{ABCD}$ formalism. However, protocols involving potentially unbounded loops (i.e., while loops) in the behaviour of agents cannot usually be modelled so that $(\mathrm{P} 2)$ and $(\mathrm{P} 4)$ hold. Fortunately, such protocols are actually rare, but considering them is one of our perspectives.

Note that our approach is compatible with the use of partial order reductions as in [23] where the main idea is that the knowledge of the intruder always grows and thus it is safe to prioritise the sending transitions with respect to receptions and local computations of agents. A simple modification of the successors functions is sufficient to achieve this.

\subsection{Proof-structure and temporal logical checking}

Many security properties such as secrecy (confidentiality), authentication, integrity, anonymity can usually be expressed only using a state-space computation since these properties only force to a reachability analysis, i.e., finding a single state that breaks one on the above properties. However, more complex property may involve distinguishing several steps in an execution and thus require to resort to temporal logics.

\subsubsection{Temporal logics}

Temporal logics have mainly two kinds of operators: logical operators and modal operators. Logical operators are the usual operators such as $\wedge, \vee$, etc. Modal operators are used to reason about time such as "until", "next-time", etc. Quantifiers can also be used to reason about paths e.g., "a formula holds on all paths starting from the current state". In LTL, one can encode formulae about the future of paths, e.g., a condition will eventually be true, a condition will be true until another fact becomes true, etc. CTL is a branching-time logic, which means that its model of time is a tree-like structure 
in which the future is not determined; there are different paths in the future, any one of which might be an actual path that is realised.

We now give the formal definition of CTL*, that subsumes both LTL and CTL. Without loss of generality, we assume that relation $T$ is total and thus all paths in $M$ are infinite. This is only a convenience to define the algorithms, but may be easily removed. We fix a set $\mathscr{A}$ of atomic propositions, which will be ranged over by $a, a^{\prime}, \cdots$. We sometimes call literals formulas of the form $a$ or $\neg a$; the set of all literals will be ranged over by $l, l_{1}, \ldots$ We use $p, p_{1}, q, \ldots$, to range over the set of state formulas and $\phi, \phi_{1}, \gamma, \ldots$, to range over the set of path formulas - both formally defined in the following. We also call path quantifiers A ("for all") and E ("exists"), and path modalities $\mathbf{X}$ ("next"), $\mathbf{U}$ ("until") and $\mathbf{R}$ ("release").

Definition 4 (Syntax of CTL*) The following grammar describes the syntax of $\mathrm{CTL}^{*}$ :

$$
\begin{gathered}
\mathscr{S}:=a|\neg a| \mathscr{S} \wedge \mathscr{S}|\mathscr{S} \vee \mathscr{S}| \mathbf{A} \mathscr{P} \mid \mathbf{E} \mathscr{P} \\
\mathscr{P}:=\mathscr{S}|\mathscr{P} \wedge \mathscr{P}| \mathscr{P} \vee \mathscr{P}|\mathbf{X} \mathscr{P}| \mathscr{P} \mathbf{U} \mid \mathscr{P} \mathbf{R} \mathscr{P}
\end{gathered}
$$

We refer to the formulas generated from $\mathscr{S}$ as state formulas and those from $\mathscr{P}$ as path formulas. We define the $\mathrm{CTL}^{*}$ formulas to be the set of state formulas.

Note that we use a particular construction on the formulas by putting the negation only adjoining to the atoms, which is a usual canonical form of $\mathrm{CTL}^{*}$ formulas that is always possible to obtain. CTL consists of those CTL* formula in which every occurrence of a path modality is immediately preceded by a path quantifier and LTL are $\mathrm{CTL}^{*}$ formula of the form $\mathbf{A} \phi$, where the only state sub-formula of $\phi$ are literals.

Definition 5 (Semantic of CTL $^{*}$ ) Let $M=(S, R, L)$ be a Kripke structure with $s \in S$ and $x$ a path in $M$. Then the satisfaction relation $\vDash$ is defined inductively as follows:

- $s \vDash a$ if $a \in L(s)$ (recall $a \in \mathscr{A})$;

- $s \vDash \neg a$ if $s \not \models a$;

- $s \vDash p_{1} \wedge p_{2}$ if $s \vDash p_{1}$ and $s \vDash p_{2}$;

- $s \vDash p_{1} \vee p_{2}$ if $s \vDash p_{1}$ or $s \vDash p_{2}$;

$-s \vDash \mathbf{A} \phi$ if for every $x \in \Pi_{M}(s), x \vDash \phi$;

$-s \vDash \mathbf{E} \phi$ if there exists $x \in \Pi_{M}(s)$ such that $x \vDash \phi$;

$-x \vDash p$ if $x(0) \vDash p$ (recall $p$ is a state formula);

$-x \vDash p_{1} \wedge p_{2}$ if $x \vDash p_{1}$ and $x \vDash p_{2}$;

$-x \vDash p_{1} \vee p_{2}$ if $x \vDash p_{1}$ and $x \vDash p_{2}$;

- $x \vDash \mathbf{X} \phi$ if $x^{1} \vDash \phi$;

- $x \vDash \phi_{1} \mathbf{U} \phi_{2}$ if there exists $i \geq 0$ such that $x^{i} \vDash \phi_{2}$ and for all $j<i, x^{j} \vDash \phi_{1}$;

$-x \vDash \phi_{1} \mathbf{R} \phi_{2}$ if for all $i \geq 0, x^{i} \vDash \phi_{2}$ or if there exists $i \geq 0$ such that $x^{i} \vDash \phi_{1}$ and for every $j \leq i, x^{j} \vDash \phi_{2}$.

The meaning of most of the constructs is straightforwards. A state satisfies $\mathbf{A} \phi$ (resp. $\mathbf{E} \phi$ ) if every path (resp. some path) starting from the state satisfies $\phi$, while a path satisfies a state formula if the initial state in the path does. $\mathbf{X}$ represents a "nexttime" operator in the usual sense of "one transition forward", while $\phi_{1} \mathbf{U} \phi_{2}$ holds of a path if $\phi_{1}$ remains true until $\phi_{2}$ becomes true. The modal operator $\mathbf{R}$ may be thought of as a "release" operator: a path satisfies $\phi_{1} \mathbf{R} \phi_{2}$ if $\phi_{2}$ remains true until both $\phi_{1}$ and 


$$
\begin{aligned}
& \begin{array}{c}
s \vdash \mathbf{A}(\Phi, \phi) \\
\begin{array}{c}
\text { true } \\
\text { if } s \vDash \phi
\end{array}
\end{array}(R 1) \quad \frac{s \vdash \mathbf{A}(\Phi, \phi)}{s \vdash \mathbf{A}(\Phi)}(R 2) \quad \frac{s \vdash \mathbf{A}\left(\Phi, \phi_{1} \vee \phi_{2}\right)}{s \vdash \mathbf{A}\left(\Phi, \phi_{1}, \phi_{2}\right)}(R 3) \quad \frac{s \vdash \mathbf{A}\left(\Phi, \phi_{1} \wedge \phi_{2}\right)}{s \vdash \mathbf{A}\left(\Phi, \phi_{1}\right) \quad s \vdash \mathbf{A}\left(\Phi, \phi_{2}\right)}(R 4) \\
& \frac{s \vdash \mathbf{A}\left(\Phi, \phi_{1} \mathbf{U} \phi_{2}\right)}{s \vdash \mathbf{A}\left(\Phi, \phi_{1}, \phi_{2}\right) \quad s \vdash A\left(\Phi, \phi_{2}, \mathbf{X}\left(\phi_{1} \mathbf{U} \phi_{2}\right)\right)}(R 5) \quad \frac{s \vdash \mathbf{A}\left(\Phi, \phi_{1} \mathbf{R} \phi_{2}\right)}{s \vdash \mathbf{A}\left(\Phi, \phi_{2}\right) \quad s \vdash \mathbf{A}\left(\Phi, \phi_{1}, \mathbf{X}\left(\phi_{1} \mathbf{R} \phi_{2}\right)\right)}(R 6) \\
& \begin{array}{c}
\frac{s \vdash \mathbf{A}\left(\mathbf{X} \phi_{1}, \ldots, \mathbf{X} \phi_{n}\right)}{s_{1} \vdash \mathbf{A}\left(\phi_{1}, \ldots, \phi_{n}\right) \quad s_{m} \vdash \mathbf{A}\left(\phi_{1}, \ldots, \phi_{n}\right)}(R 7) \\
\text { if } \operatorname{succ}(s)=\left\{s_{1}, \ldots, s_{m}\right\}
\end{array}
\end{aligned}
$$

Fig. 3 Proof rules for LTL checking [7].

$\phi_{2}$ ( $\phi_{1}$ releases the path from the obligations) or $\phi_{2}$ is always true. For two examples of security properties:

1. Fairness is a CTL formula: $\mathbf{A G}\left(\operatorname{recv}\left(c_{1}, d_{2}\right) \Rightarrow \mathbf{E F} \operatorname{recv}\left(c_{2}, d_{1}\right)\right)$ if we assume two agents $c_{1}$ and $c_{2}$ that possess items $d_{1}$ and $d_{2}$, respectively, and wish to exchange them; it asserts that if $c_{1}$ receives $d_{2}$, then $c_{2}$ has always a way to receive $d_{1}$.

2. The availability of an agent can be a LTL formula that requires that all the messages $m$ received by this agent $a$ will be processed eventually, which can be formalised as: $\mathbf{A G}(\operatorname{rcvd}(a, m) \Rightarrow(\mathbf{F} \neg \operatorname{rcvd}(a, m)))$

where the two syntaxic sugars are: (1) $\mathbf{G}(p)$ is for "globally" and is equal to false $\mathbf{R} p$; (2) $\mathbf{F}(p)$ is for "finally" and is equal to true $\mathbf{U} p$.

\subsubsection{Checking a LTL formula}

In [7], the authors give an efficient algorithm for model-checking LTL then CTL ${ }^{*}$ formula. The algorithm is based on a collection of top-down proof rules for inferring when a state in a Kripke structure satisfies a LTL formula. It is close to a Tableau method [25]. These rules are reproduced in Fig. 3, they work on assertions of the form $s \vdash \mathbf{A} \Phi$ where $s \in S$ and $\Phi$ is a set of path formula.

Semantically, $s \vdash \mathbf{A} \Phi$ holds if $s \vDash \mathbf{A}\left(\bigvee_{\phi \in \Phi} \phi\right)$. We write $\mathbf{A}\left(\Phi, \phi_{1}, \cdots, \phi_{n}\right)$ to represent $\mathbf{A}\left(\Phi \cup\left\{\phi_{1}, \cdots, \phi_{n}\right\}\right)$ and we consider $\mathbf{A}(\emptyset)=\emptyset$. If $\sigma$ is an assertion of the form $s \vdash \mathbf{A} \Phi$ then we use $\phi \in \sigma$ to denote that $\phi \in \Phi$. We may also drop $\mathbf{A}$ and write $s \vdash \Phi$ for an assertion if the context allows it.

Definition 6 (Proof structure [7]) Let $\Sigma$ be a set of nodes, $\Sigma^{\prime} \stackrel{\text { df }}{=} \Sigma \cup$ true, $V \subseteq \Sigma^{\prime}$, $E \subseteq V \times V$ and $\sigma \in V$. Then $\langle V, E\rangle$ is a proof structure for $\sigma$ if it is a maximal directed graph such that for every $\sigma^{\prime} \in V, \sigma^{\prime}$ is reachable from $\sigma$, and the set $\left\{\sigma^{\prime \prime} \mid\left(\sigma^{\prime}, \sigma^{\prime \prime}\right) \in\right.$ $E\}$ is the result of applying some rule to $\sigma^{\prime}$.

Intuitively, a proof structure for $\sigma$ is a direct graph that is intended to represent an (attempted) "proof" of $\sigma$. In what follows, we consider such a structure as a directed graph and use traditional graph notations for it. Note that in contrast with traditional definitions of proofs, proof structures may contain cycles. In order to define when a proof structure represents a valid proof of $\sigma$, we use the following notion: 
Definition 7 (Successful proof structure [7]) Let $\langle V, E\rangle$ be a proof structure.

$-\sigma \in V$ is a leaf iff there is no $\sigma^{\prime}$ such that $\left(\sigma, \sigma^{\prime}\right) \in E$. $\sigma$ is successful iff $\sigma \equiv$ true.

- An infinite path $\pi=\left\langle\sigma_{0}, \sigma_{1}, \cdots\right\rangle$ in $\langle V, E\rangle$ is successful iff some assertion $\sigma_{i}$ infinitely repeated in $\pi$ satisfies the following: there exists $\phi_{1} \mathbf{R} \phi_{2} \in \sigma_{i}$ such that for all $j \geq i, \phi_{2} \notin \sigma_{j}$.

- $\langle V, E\rangle$ is partially successful iff every leaf is successful. $\langle V, E\rangle$ is successful iff it is partially successful and each of its infinite paths is successful.

Roughly speaking, an infinite path is successful if at some point a formula of the form $\phi_{1} \mathbf{R} \phi_{2}$ is repeatedly "regenerated" by application of rule R6, i.e., the right-hand sub-goal of this rule application appears each time on the path. Note that after $\phi_{1} \mathbf{R} \phi_{2}$ occurs on the path, $\phi_{2}$ should not, because, intuitively, if $\phi_{2}$ was true then the success of the path would not depend on $\phi_{1} \mathbf{R} \phi_{2}$, while if it was false then $\phi_{1} \mathbf{R} \phi_{2}$ would not hold. Note also that if no rule can be applied (i.e., $\Phi=\emptyset$ ) then the proof-structure is unsuccessful and thus the formula does not hold. We now have the following result:

Theorem 1 (Proof-structure and LTL [7]) Let $M$ be a Kripke structure with $s \in S$ and $\mathbf{A} \phi$ an LTL formula, and let $\langle V, E\rangle$ be a proof-structure for $s \vdash \mathbf{A}\{\phi\}$. Then $s \vDash \mathbf{A} \phi$ iff $\langle V, E\rangle$ is successful.

One consequence of this theorem is that if $\sigma$ has a successful proof-structure, then all proof-structures for $\sigma$ are successful. Thus, it turns out that the success of a finite proof-structure may be determined by looking at its strongly connected components (SCCs, we recall that a SCC of a directed graph is a maximal component in which every vertex can be reached from every other) or any accepting cycle. The efficient algorithm of [7] (described later) combines the construction of a proof-structure with the process of checking whether the proof-structure is successful using a Tarjan like algorithm for SCC computation (and a recursive decomposition of a $\mathrm{CTL}^{*}$ formula into several LTL formula) but a NDFS [28] one could be used equally.

Call a SCC $\mathscr{O}$ of $\langle V, E\rangle$ nontrivial if there exist (not necessary distinct) $v, v^{\prime} \in \mathscr{O}$ such that there is a path containing a least one edge from $v$ to $v^{\prime}$. For any $V^{\prime} \subseteq V$ we may define the success set of $V^{\prime}$ as follows:

$$
\operatorname{Success}\left(V^{\prime}\right) \stackrel{\text { df }}{=}\left\{\phi_{1} \mathbf{R} \phi_{2} \mid \exists \sigma \in V^{\prime}: \phi_{1} \mathbf{R} \phi_{2} \in \sigma \text { and } \forall \sigma^{\prime} \in V^{\prime}: \phi_{2} \notin \sigma^{\prime}\right\} .
$$

We say that $V^{\prime}$ is successful if and only if $\operatorname{Success}\left(V^{\prime}\right) \neq \emptyset$ we have the following:

Theorem 2 (SCC and LTL [7]) A partially successful proof structure $\langle V, E\rangle$ is successful if and only if every nontrivial SCC of $\langle V, E\rangle$ is successful.

For example, Fig. 4 gives the successful proof-structure of the checking of $s_{1} \vDash$ $\mathbf{A}(b \mathbf{R} a)$ for a Kripke structure with three states such that atomic proposition $a$ is always true and $b$ is only true for state $s_{2}$.

\section{BSP state-space construction of security protocols}

Based on the properties defined in section 2.2.3, we have designed, in an incremental manner, a BSP algorithms for efficiently computing the state-space of security protocols. In order to explain our parallel algorithm, we start with a generic and sequential 

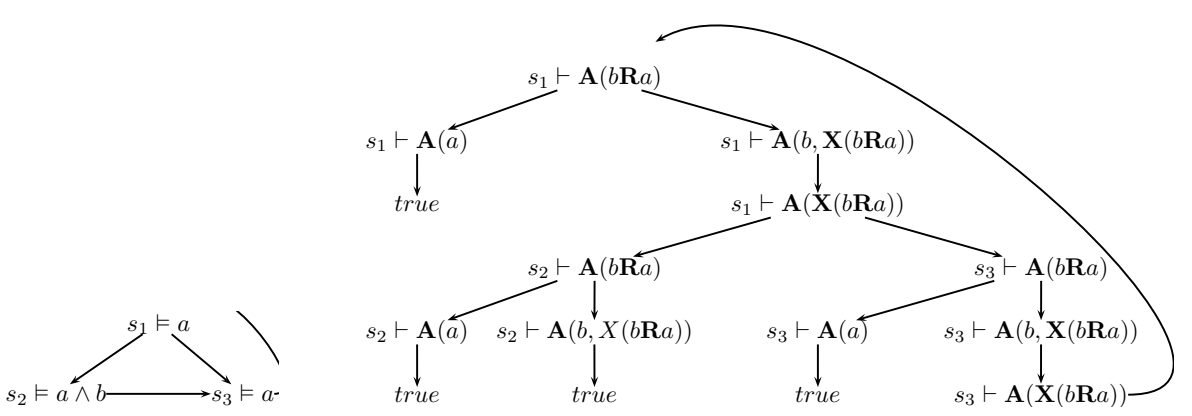

Fig. 4 Proof-structure (right) of $s_{1} \vdash \mathbf{A}(b \mathbf{R} a)$ for a simple Kripke structure (left).

algorithm that corresponds to the usual construction of a state-space and we also give a generic (in the sence of independant of succ) parallel algorithm for state-space computing which will the basis for the parallel version. Successive improvements will result in a parallel algorithm that remains quite simple in its expression but that actually relies on a precise use of a consistent set of observations and algorithmic modifications. We will show that this algorithm is efficient despite its simplicity.

\subsection{Usual generic sequential algorithm}

The algorithm given in 5 involves a set todo of states that is used to hold all the states whose successors have not been constructed yet; initially, it contains only the initial state $\mathrm{s}_{0}$. Then, each state $\mathrm{s}$ from todo (taken using the pick routine) is processed in turn and added to a set known while its successors are added to todo unless they are known already. At the end of the computation, known holds all the states reachable from $\mathrm{s}_{0}$, that is, the state-space $S$. Note that this algorithm could be made strictly depth-first by using todo as a stack, and breadth-first by using todo as a fifo queue. This has not been considered here.

We now show how the sequential algorithm can be parallelised in BSP and how several successive improvements can be introduced.

\subsection{A naive and generic BSP algorithm for state-space computation}

One of the main technical issues in the distributed-memory state-space construction is to partition the state-space among the participating machines. Most of approaches

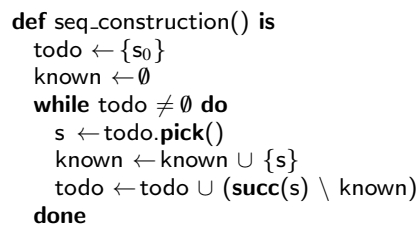

Fig. 5 Sequential construction of the state-space. 
exchanged states - it is mainly the MPI's alltoall primitive. Notice that, by postponing communication, this function allows buffered sending and forbids sending several times the same state. More formally, at processor mypid:

$$
\text { BspExchange }(\text { tosend })=\left\{\begin{array}{l}
\text { total }=\sum_{k=0}^{\text {nprocs }-1} \sum_{i=0}^{\text {nprocs }-1} \mid \text { tosend }[[k]][i] \mid \\
\operatorname{rcv}=\bigcup_{i=0}^{\text {nprocs-1 }} \text { tosend }[[i]][\text { mypid }]
\end{array}\right.
$$

where tosend $[i]]$ represents the array tosend at processor $i$.

In order to terminate the algorithm, we use the additional variable total in which we count the total number of sent states i.e., total is an upper sum of the sizes of all the sets todo after the synchronisation. We have thus not used any complicated methods as the ones presented in [24]. It can be noted that the value of total may be greater than the total number of states in the todo sets. Indeed, it may happen that two processors compute a same state owned by a third processor, in which case two states are exchanged but only one is kept upon reception. Moreover, if this state has been also computed by its owner, it will be ignored. This not a problem in practise because in the next super-step, this duplicated count will disappear. In the worst case, the termination requires one more super-step during which all the processors will process an empty todo, resulting in an empty exchange and thus total $=0$ on every processor, yielding the termination.

We now consider how to incrementally optimise this BSP algorithm for the case of security protocols using their specific properties. An interesting point of this work is that the main loop of the BSP algorithm will be kept unchanged, i.e., only functions local_successors and exchange will be modified.

3.3 Dedicated BSP algorithm for state-space construction of security protocols

\subsubsection{Increasing local computation time}

Using the above naive parallel algorithm, function cpu distributes evenly the states over the processors. However, each super-step is likely to compute very few states because only too few computed successors are locally owned. This results in a bad balance of the time spent in computation with respect to the time spent in communication. If more states can be computed locally, this balance improves but also the total communication time decreases because more states are computed during each call to function local_successors. 


\subsubsection{Decreasing local storage}

One can observe that the structure of the computation now matches the structure of the protocol execution: each super-step computes the executions of the protocol until a message is received. As a consequence, from the states exchanged at the end of a super-step, it is not possible to reach states computed in any previous super-step. This corresponds to property (P2).

This kind of progression in a model execution is the basis of the sweep-line method [14] that aims at reducing the memory footstep of a state-space computation by exploring states in an order compatible with progression. It thus becomes possible to regularly dump from the main memory all the states that cannot be reached anymore - a disk-based backup can also be made if it is necessary to restore the trace of a forbidden computation. Thus, in Fig. 8, statement dump(known) resets known to an empty set, possibly saving its content to disk if this is desirable. The rest of function exchange is simplified accordingly.

Enforcing such an exploration order is usually made by defining on states a measure of progression slice as stated in property (P2). In our case however, such a measure is not needed explicitly because of the match between the protocol progression and the super-steps succession. So we can apply the sweep-line method by making a simple modification of the exploration algorithm. This algorithm is as before except that we empty known at the end of each super-step, just before the next one. The corresponding new function exchange is given at the top-right of Fig. 8.

\subsubsection{Balancing the Computations}

During our benchmark, we have found that using $\mathrm{cpu}_{\mathscr{R}}$ can introduce a bad balance of the computations due to a lack of information when hashing only on $\mathscr{R}$. Thus, the final optimisation step aims at rebalancing the workload. To do so, we exploit the following observation: for all the protocols we have studied so far, the number of computed states during a super-step is usually closely related (proportional actually) to the number of states received at the beginning of the super-step. So, before to exchange the states themselves, we can first exchange information about how many states each processor has to send and how they will be spread onto the other processors. Using this information, we can anticipate and compensate balancing problems.

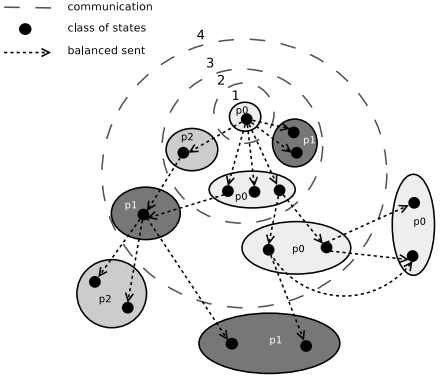

Fig. 9 Distribution of the sets of states.

To compute the balancing information, we use a new partition function $\mathrm{Cpu}_{B}$ that is equivalent to $\mathrm{cpu}_{\mathscr{R}}$ without modulo. This function defines classes of states for which $\mathrm{cpu}_{B}$ returns the same value. Those classes are like "bag-of-tasks" [31] that can be distributed over the processors independently, see Fig. 9. To do so, we compute a histogram of these classes on each processor, which summarises how $\mathrm{cpu}_{\mathscr{R}}$ would dispatch the states. This local histograms are then exchanged, yielding a global histogram that is exploited to compute on each processor a better dispatching of the states it has to send. This is made 
by placing the classes according to a simple heuristic for the bin packing problem: the largest class is placed onto the less charged processor, which is repeated until all the classes have been placed. It is worth noting that this placement is computed with respect to the global histogram, but then, each processor dispatches only the states it actually holds, using this global placement. Moreover, if several processors compute a same state, these identical states will be in the same class and so every processor that holds such states will send them to the same target. So there is no possibility of duplicated computation. We call this algorithm "Balance".

These operations are detailed in the bottom-right part of Fig 8, where variables histoL and histoG store respectively the local and global histograms, and function BinPack implements the dispatching method described above. In function balance, $\sharp X$ denotes the cardinality of set $X$. Function BspMulticast is used to allow each processor to send its local histogram to every processor and receive in turn their histograms, allowing to build the global one. It thus involves a synchronisation barrier.

It may be remarked that the global histogram is not fully accurate since several processors may have a same state to be sent. Nor the computed dispatching is optimal since we do not want to solve a NP-hard bin packing problem. But, as shown in our benchmarks below, the result is yet fully satisfactory. Finally, it is worth noting that if a state found in a previous super-step may be computed again, it would be necessary to known which processor owns it: this could not be obtained efficiently when dynamic remapping is used. But that could not happen thanks to our sweep-line compatible exploration order. Our dynamic states remapping is thus correct because states classes match the locality of computation.

\subsection{Experimental results}

In order to evaluate our algorithms, we have implemented a prototype version in PYTHON, using SNAKES for the Petri net part (which also allowed for a quick modelling of the protocols, including the inference rules of the Dolev-Yao attacker) and a BSP-PYTHON library [27] for the BSP routines (which are close to a MPI's "alltoall"). We actually used the MPI version (with mpich) of the BSP-PYTHON library. While largely suboptimal (PYTHON programs are interpreted and there is no optimisation about the representation or computation of the states in SNAKES), this prototype nevertheless allows and accurate comparison of the various algorithms - execution times of PYTHON programs are very stable over several execution and not depend of code placement in the main memory or of unpredictable underlying optimizations of the compiler/processor. The benchmarks presented below have been performed using the cluster of the first author's laboratory that is 20 PCs connected through a 1 Gigabyte Ethernet network; Each PC is equipped with a 2 Ghz Intel $($ Pentium $($ ) dual core CPU, with $2 \mathrm{~GB}$ of physical memory; This allowed to simulate easily a BSP computer with at most 40 processors equipped with $1 \mathrm{~GB}$ of memory each.

Our cases study involved the following five protocols: (1) Needham-Schroeder (NS) public key protocol for mutual authentication; (2) Yahalom (Y) key distribution and mutual authentication using a trusted third party; (3) Otway-Rees (OR) key sharing using a trusted third party; (4) Woo and Lam Pi (WLP) authentification protocol 
with public keys and trusted server; (5) Kao-Chow (KC) key distribution and authentication. All are documented at the Security Protocols Open Repository (SPORE) (http://www.lsv.ens-cachan.fr/Software/spore).

For each protocol, we have built a modular model allowing for defining easily various scenarios involving different numbers of each kind of agents. We note our scenarios NS $x-y$ indicating $x$ instances of Alice and $y$ instances of Bob with one unique sequential session; Y (resp. OR, KC, WLP)-x-y-z n indicating $x$ instances of the Server, $y$ of Alice, $z$ of Bob, involved in $n$ sequential sessions.

We give the total time of computation and note SWAP when at least one processor has started to swap to disk due to a lack of main memory for storing its part of the state-space. We also note COMM when a similar situation happens during communication: the system is unable to received data since not enough memory is available. We also give the number of states. For the Needham-Schroeder protocol, we have:

\begin{tabular}{|l|l|l|l|}
\hline Scenario & Naive & Balance & Nb_states \\
\hline NS _1-2 & $0 \mathrm{~m} 50.222 \mathrm{~s}$ & $0 \mathrm{~m} 42.095 \mathrm{~s}$ & 7807 \\
\hline NS _1-3 & $115 \mathrm{~m} 46.867 \mathrm{~s}$ & $61 \mathrm{~m} 49.369 \mathrm{~s}$ & 530713 \\
\hline NS _2-2 & $112 \mathrm{~m} 10.206 \mathrm{~s}$ & $60 \mathrm{~m} 30.954 \mathrm{~s}$ & 456135 \\
\hline
\end{tabular}

For the Yahalom protocol:

\begin{tabular}{|l|l|l|l|}
\hline Scenario & Naive & Balance & Nb_states \\
\hline $\mathrm{Y} \_1-3-1$ & $12 \mathrm{~m} 44.915 \mathrm{~s}$ & $7 \mathrm{~m} 30.977 \mathrm{~s}$ & 399758 \\
\hline $\mathrm{Y} \_1-3-1 \_2$ & $30 \mathrm{~m} 56.180 \mathrm{~s}$ & $14 \mathrm{~m} 41.756 \mathrm{~s}$ & 628670 \\
\hline $\mathrm{Y} \_1-3-1 \_3$ & $481 \mathrm{~m} 41.811 \mathrm{~s}$ & $25 \mathrm{~m} 54.742 \mathrm{~s}$ & 931598 \\
\hline $\mathrm{Y} \_2-2-1$ & $2 \mathrm{~m} 34.602 \mathrm{~s}$ & $2 \mathrm{~m} 25.777 \mathrm{~s}$ & 99276 \\
\hline $\mathrm{Y} \_3-2-1$ & COMM & $62 \mathrm{~m} 56.410 \mathrm{~s}$ & 382695 \\
\hline $\mathrm{Y} \_2-2-2$ & $2 \mathrm{~m} 1.774 \mathrm{~s}$ & $1 \mathrm{~m} 47.305 \mathrm{~s}$ & 67937 \\
\hline
\end{tabular}

For the Otway-Rees protocol:

\begin{tabular}{|l|l|l|l|}
\hline Scenario & Naive & Balance & Nb_states \\
\hline OR_1-1-2 & $38 \mathrm{~m} 32.556 \mathrm{~s}$ & $24 \mathrm{~m} 46.386 \mathrm{~s}$ & 12785 \\
\hline OR_1-1-2_2 & $196 \mathrm{~m} 31.329 \mathrm{~s}$ & $119 \mathrm{~m} 52.000 \mathrm{~s}$ & 17957 \\
\hline OR_1-1-2_3 & $411 \mathrm{~m} 49.876 \mathrm{~s}$ & $264 \mathrm{~m} 54.832 \mathrm{~s}$ & 22218 \\
\hline OR_1-2-1 & $21 \mathrm{~m} 43.700 \mathrm{~s}$ & $9 \mathrm{~m} 37.641 \mathrm{~s}$ & 1479 \\
\hline
\end{tabular}

For the Woo and Lam Pi protocol:

\begin{tabular}{|l|l|l|l|}
\hline Scenario & Naive & Balance & Nb_states \\
\hline WLP _1-1-1 & $0 \mathrm{~m} 12.422 \mathrm{~s}$ & $0 \mathrm{~m} 9.220 \mathrm{~s}$ & 4063 \\
\hline WLP _1-1-1_2 & $1 \mathrm{~m} 15.913 \mathrm{~s}$ & $1 \mathrm{~m} 1.850 \mathrm{~s}$ & 84654 \\
\hline WLP _1-1-1_3 & COMM & $24 \mathrm{~m} 7.302 \mathrm{~s}$ & 785446 \\
\hline WLP _1-2-1 & $2 \mathrm{~m} 38.285 \mathrm{~s}$ & $1 \mathrm{~m} 48.463 \mathrm{~s}$ & 95287 \\
\hline WLP _1-2-1_2 & SWAP & $55 \mathrm{~m} 1.360 \mathrm{~s}$ & 946983 \\
\hline
\end{tabular}

For the Kao-Chow protocol:

\begin{tabular}{|l|l|l|l|}
\hline Scenario & Naive & Balance & Nb_states \\
\hline KC_1-1-1 & $4 \mathrm{~m} 46.631 \mathrm{~s}$ & $1 \mathrm{~m} 15.332 \mathrm{~s}$ & 376 \\
\hline KC_1-1-2 & $80 \mathrm{~m} 57.530 \mathrm{~s}$ & $37 \mathrm{~m} 50.530 \mathrm{~s}$ & 1545 \\
\hline KC_1-1-3 & $716 \mathrm{~m} 42.037 \mathrm{~s}$ & $413 \mathrm{~m} 37.728 \mathrm{~s}$ & 4178 \\
\hline KC_1-1-1_2 & $225 \mathrm{~m} 13.406 \mathrm{~s}$ & $95 \mathrm{~m} 0.693 \mathrm{~s}$ & 1163 \\
\hline KC_1-2-1 & $268 \mathrm{~m} 36.640 \mathrm{~s}$ & $159 \mathrm{~m} 28.823 \mathrm{~s}$ & 4825 \\
\hline
\end{tabular}

We can see that the overall performance of our dedicated "Balance" algorithm is always very good compared to the naive and general one. This holds for large statespaces as well as for smaller ones. Furthermore, the naive implementation can swap, which never happens for the "Balance" one. 
By measuring the memory consumption of our "Balance" algorithm, we could confirm the benefits of our sweep-line implementation when large state-spaces are computed. For instance, in a NS scenario with 5M states, we observed an improvement of the peak memory usage from $97 \%$ to $40 \%$ (maximum among all the processors). Similarly, for a Y scenario with $1 \mathrm{M}$ states, the peak decreases from $97 \%$ to $60 \%$ (states in Y use more memory that states in NS). Similarly, for the WLP_1-2-1_2, the peak decreases so that the computation does not swap. For Y_3-2-1, "Balance" used a little less memory but this is enough to avoid crashing the whole machine. We also observed, on very large state-spaces, that the naive implementation exhausts all the available memory and some processors start to use the swap, which causes a huge performance drop. This never happened using our sweep-line implementation.

As a last observation about our algorithm, we would like to emphasise that we observed a linear speedup with respect to the number of processors. In general, most parallel algorithms suffer from an amortised speedup when the number of processors increases. This is almost always caused by the increasing amount of communication that becomes dominant over the computation. Because our algorithm is specifically dedicated to reduce the number of cross transitions, and thus the amount of communication, this problem is largely alleviated and we could observe amortised speedup only for very small models (less than 100 states) for which the degree of intrinsic parallelism is very reduced but whose state-space is in any way computed very quickly.

\section{BSP on-the-fly LTL checking of security protocols}

\subsection{A sequential imperative algorithm for generic on-the-fly LTL checking}

[7] gives a recursive algorithm for LTL checking. It is mainly the recursive Tarjan algorithm for a SCC decomposition but working on proof-structures and finding onthe-fly a successful SCC to validate or not the formula: it combines the construction of a proof-structure with the process of checking whether it is successful; as soon as it is determined that the partially constructed structure cannot be extended successfully, the routine halts the construction of the structure and returns anwser False.

To be close to our previous distributed algorithms, we have chosen to derecursify this algorithm using, as usual, an explicit stack to record the recursive calls. Instead of the recursive procedure, we use procedures call_Itl, loop_Itl, up_lt $\mid$ and ret_Itl and an additional stack todo (which contains initially the initial state) to achieve a derecursification of the traditional recursive Tarjan's algorithm. Note the definition of subroutines in the main procedure without their body which are given separately. This notation is used to define the scope of variables and to decompose the algorithm into several routines. Fig. 10 gives this algorithm which operates as follows.

Roughly speaking, a break of the procedure loop_ltl resumes the nested exploration by popping the stack todo in which we have placed the next state to explore. The backtracking is done by the procedure ret_|t| which restores the control to its parent call, that in turn may possibly resume the exploration of its children.

Additional informations are stored in each assertion (a vertex) $\sigma$ of the proofstructure that enable the detection of unsuccessful SCC. We use an implicit mapping 


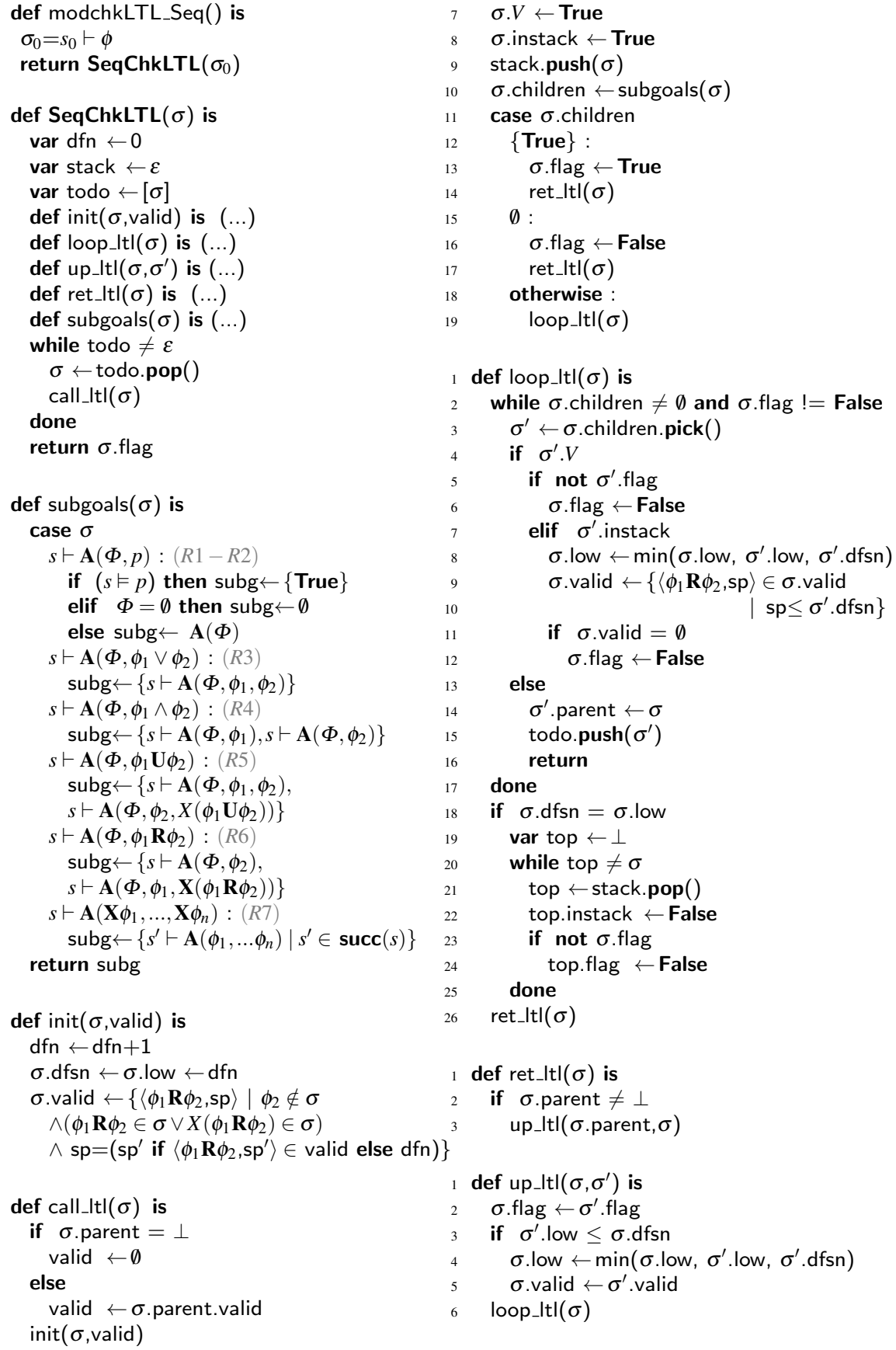

Fig. 10 Sequential imperative algorithm for LTL model checking. 
from the pairs $\langle$ state, $\mathbf{A} \Phi\rangle$ (as keys) to fields of assertions that are assigned appropriately when assertions are first visited. These fields are the following:

- The algorithm of [7] maintains two sets of assertions: V (for visited), which records the assertions that have been encountered so far, and $F$, which contains assertions that have been determined to be False (by abuse of language, we say that the anwser of the assertion is invalid). To implement this, $\sigma$ has two boolean fields .V (initially False) and .flag. The latter determines the validity of the assertion if $\sigma . V$ is true. Initially flag is True, and it becomes False either if the set of subgoals of an assertion is empty or if one of these two conditions is satisfied:

- one of the subgoals of the assertion is already visited and its flag is False (this case will actually occur when we will check CTL* formulas);

- an unsuccessful nontrivial strongly component is found by testing if the set valid is empty or not.

- The field .parent is a set of assertions $\sigma^{\prime}$ such that $\left(\sigma^{\prime}, \sigma\right) \in E$ that is there is a edge from $\sigma^{\prime}$ to $\sigma$ in the proof-structure (direct graph); it is mainly used for backtracking the results of nested computations; in the same manner, the field .children is also a set of assertions such that $\left(\sigma, \sigma^{\prime}\right) \in E$.

- As the algorithm consists of a depth-first exploration of the proof-structure, $\sigma$ has two specific fields used to detect SCCs: .dfsn (the depth-first search number of $\sigma$ ) and .low (the record of the dept-first search number of the "oldest" ancestor of $\sigma$ that is reachable from $\sigma$ ), both expressing respectively the depth-first search number (by incrementation of the dfn variable) and the smallest depth-first search number of a state that is reachable from the considered state. The detection that a state belongs to a SCC is made by testing if a successor is in the global stack. A SCC is found at a certain point if at the end of a some course of the proof-structure, the field .low coincides with the field .dfsn.

- We associate the field .valid which is the set of pairs of the form $\left\langle\phi_{1} \mathbf{R} \phi_{2}, \mathrm{sp}\right\rangle$. Intuitively, the formula component of such a pair may be used as evidence of the success of the SCC that $\sigma$ might be in, while sp records the "starting point" of the formula, i.e., the depth-first number of the assertion in which this occurrence of the formula first appeared.

- We also need a test of membership of assertions in the global stack. In order to have a constant time test avoiding to actually explore the stack, we add another field stack that is a Boolean answering whether the assertion in the stack or not.

For model-checking LTL formulas, we begin by the procedure modchkLTL_Seq which initiates the variables dfn and stack and start the depth-first exploration by putting the initial assertion in todo (lines 6-13). The main loop over todo is to construct a successful proof structure (lines 14-17).

Procedure call_Itl proceeds as follows. The successors of the current assertion are computed by subroutine subgoals (line 10): it applies the rules of Fig. 3 and when no subgoal is found an error occurs (this is an unsuccessful proof structure). If the children (subgoals) of $\sigma$ are all True (valid) then it backtracks to the parent call (using procedure ret_lt). Else there is no child and thus it is an unsuccessful proof structure, the assertion is not-valid and it again backtracks to the parent call. Otherwise, we need to iterate over the children using a call to loop_ltl. 
Procedure loop_Itl proceeds as follow. If subgoal $\sigma^{\prime}$ has already been examined (i.e., field $V$ is true in line 4) and found to be False (line 5) then the proof structure cannod be successul, and we terminate the processing in order to return False: we pop all the assertions from the stack and if they are in the same SCC, they are marked to be False (lines 19-23). If $\sigma^{\prime}$ has not been found False, and if $\sigma^{\prime}$ is in the stack (meaning that its SCC is still being constructed), the $\sigma$ and $\sigma^{\prime}$ will be in the same SCC: we reflect this by updating $\sigma$.low accordingly. We also update $\sigma$.valid by removing formulas whose starting points occur after $\sigma^{\prime}$; as we show below, these formulas cannot be used as evidence for the success of the SCC containing $\sigma$ and $\sigma^{\prime}$ (lines 814). Once the subgoal processing is completed, loop_ltl checks to see whether a new SCC component has been detected; if no, it removes it from the stack (lines 18-23) and finally backtracks to the parent call (line 25).

Procedure ret_Itl is just a call to up_Itl if the assertion has no "parent". Procedure up_It update the field .low and .dfsn as the traditional Tarjan algorithm and restarts the exploration of the other children by a call to loop_|tl.

Notice that using "proof-structures" is not common, LTL checking is traditionally perform by the test of emptiness of a Büchi automaton which is the product of the LTS and of the formula translated to an automaton. Generally, a NDFS algorithm checks the presence of an accepting cycle. Our approach "simplifies" the use of our two successors functions and allows us to check $\mathrm{CTL}^{*}$ formula without using any (alternative hesitant) automaton which are slow to compute.

\subsection{BSP on-the-fly checking a LTL formula over security protocols}

As explained in the previous sections, we use two LTS successor functions for constructing the Kripke structure: succ $\mathscr{R}_{\mathscr{R}}$ ensures a measure of progression "slice" that intuitively decomposes the Kripke structure into a sequence of slices $S_{0}, \ldots, S_{n}$ where transitions from states of $S_{i}$ to states of $S_{i+1}$ come only from succ $\mathscr{R}$ and there is no possible path from states of $S_{j}$ to states $S_{i}$ for all $i<j$. In this way, we have used a distribution of the Kripke structure across the processors using the $\mathrm{cpu}_{B}$ function, we thus naturally extend this function to assertions $\sigma$ using only the state field. Then, with this distribution, the only possible accepting cycles or SCCs are local to each processor. Thus, because proof-structures follow the Kripke structure (rule R7), accepting cycles or SCCs are also only locals. Call this sequential algorithm SeqChkLTL (the only difference with the previous one is the subprocedure subgoal due to the two successors functions) which takes an assertion $\sigma \equiv s \vdash \mathbf{A} \Phi$. It also modifies the set of assertions to be sent (for the next super-step). Now, we can design our BSP algorithm which is mainly an iteration over the independent slices, one slice per super-step and, on each processor, working on independent sub-parts of the slice by calling SeqChkLTL. This SPMD (this executed by each processor executes) algorithm is given in Fig. 11.

The main procedure ParChkLTL first initialises so that one processor owns the initial assertion and saves it in its todo list. The variable total stores the number of states to be processed at the beginning of each super-step; $V$ and $E$ store the proofstructure (in fact we manipulate an implicit mapping of assertions through the fields 

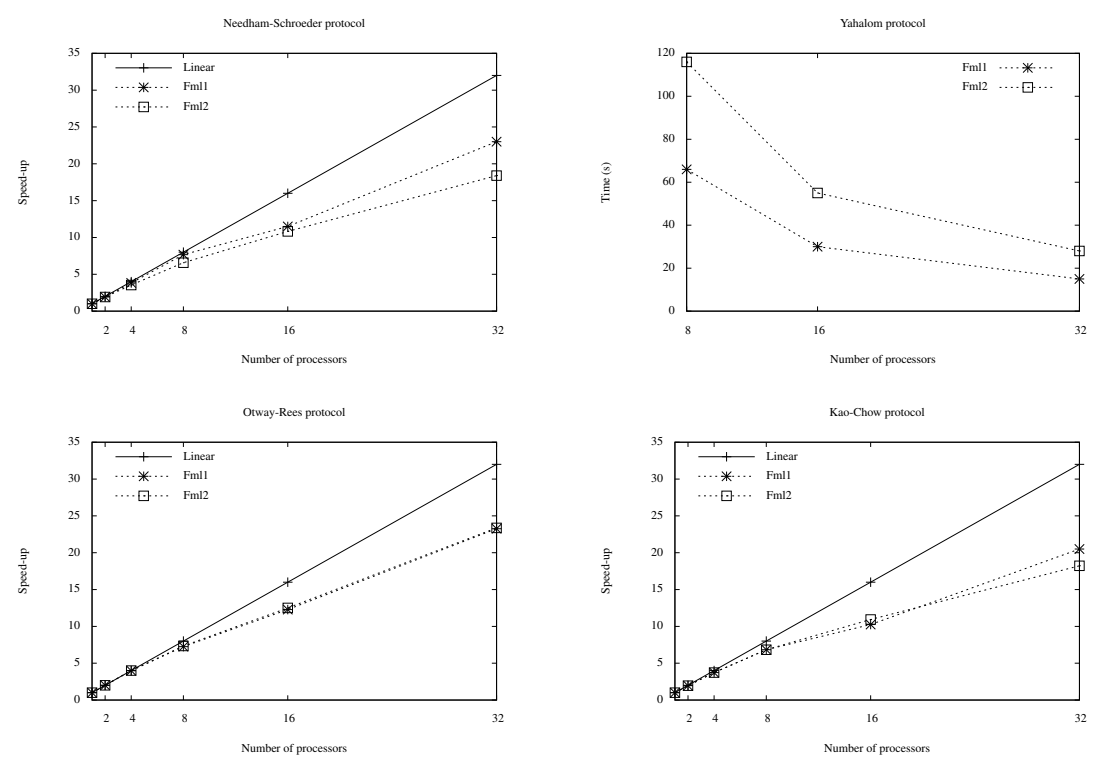

Fig. 13 Benchmark results of our BSP algorithm for LTL checking apply to four protocols where Fml1 is "secrecy" and Fml2 is "availability".

search while the sequential exploration is depth-first, which usually succeeds earlier. But when all the exploration has to be performed, which is widely acknowledged as the hardest case, our algorithm is always much faster. Moreover, we sometimes could not compute the state-space sequentially while the distributed version succeeded.

Fig. 13 gives the speed-up for each of the two formulas and two sessions of each protocol. For the Yahalom protocol, the computation fails due to a lack of main memory if less that four nodes are used: so we could not give the speedup but only execution times. We observed a relative speed-up with respect to the number of processors.

\section{BSP on-the-fly CTL* checking of security protocols}

As for LTL, we first present a sequential algorithm and then specialised parallel algorithms for security protocols. The first parallel algorithm called "naive" is a first attempt to extend the parallel algorithm for LTL checking to $\mathrm{CTL}^{*}$ formulas whereas the second one optimises the communications and reduces the number of super-steps.

\subsection{A sequential algorithm for CTL* checking}

The algorithm of [7] (named SeqRecChkCTL* and presented in Fig. 14) processes a formulae $P$ either by recursively call SeqChkLTL appropriately when it encounters assertions of the form $s \vdash \mathbf{A} \Phi$ or $s \vdash \mathbf{E} \Phi$, or by decomposing the formulae $P$ into sub-formulas. Note the use of an equivalence of an exits-formula with the negation of the corresponding forall-formula to check the latter. 


\subsubsection{General idea}

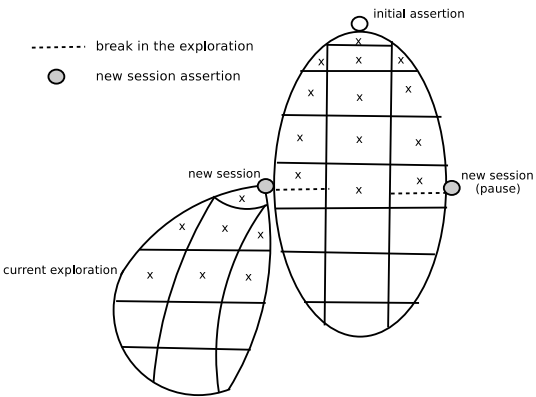

Fig. 15 Naive generation of the LTL sesssions.

The algorithm works as follow. A first step is to recursively decomposing the formulae for finding the first assertion of the form $s \vDash \mathbf{A}(\phi)$. Then a main loop is used to proceed the received assertions: for each of them, an exploration is used to decompose the formulae and run SeqChkCTL* adequately to check for an unsuccessful SCC in the proof-structure. Recall that we name this computation a "LTL session" and considering it as a distinguished object. During the generation of the proof-structure, when a sub-formulae beginning by $\mathbf{A}$ or $\mathbf{E}$ is found (case of rules $R 1$ and $R 2$ ), the ongoing session is paused (see Fig. 15), pushed onto a stack of waiting sessions and is kept their until the result of a new session to check the validity of $s \vDash p$ is available.

There are three main problems. (1) different processors can throw sessions. (2) a session can induce several super-steps (slices) if it is a path formula. This is due to the double recursion of the $\mathrm{CTL}^{*}$ checking. (3) the different sessions are not fully disjoints: states of the Kripke structures as well as assertions can be shared, that happens when the same sub-parts of the Kripke structure are generated and when sets of formula in the assertions are not disjoints. This results in an embarrassingly parallel computation on this set of sessions. A naive solution is to globally select one of these generated sessions (the other still remain in a distributed global stack) and to entirely compute this session until another session is thrown or an answer is found (validity of a $s \vDash p$ ). A part of this algorithm called ParNaiveChkCTL* is given in Fig. 16 and the full algorithm is available in [26].

\subsubsection{Main loop}

The initial recursive decomposition is performed in lines 9-23. Then, calls to the main loop main_ParNaive* are performed in lines 21 and 23. The following variables are used during the computation of the main loop:

- out_stack (initially empty) manages the exploration "depth" of the sessions by storing the LTL sessions;

- answer_ttl saves the answer/validity (True or False) when a session is finished;

- flag_list contains the assertions infringing the computation and is used for the backtracking;

- mck represents the session currently used (exploration, recovery, backtracking).

The main loop proceeds, until the stack of sessions is empty, by creating a session for the assertion if it is new (line 30); then by performing the exploration (.explore) and by pushing this session at the top of the stack. The flags are assertions that are not valid for the session (which does not induces that the overall formula is invalid if the 
a straightforward modification of subgoal by returning an empty set of successors and a flag that indicates if this is due to an invalid formulae or to the need for pausing the current computation. In the latter case, the ongoing "session" is paused and is set waiting for the answer of the new session based on the appropriate assertion.

Finally, method .recovery resumes a paused computation by passing as an argument the flag value corresponding to the validity of the assertion previously returned - and awaiting to test. This flag is an answer corresponding of the validity required on the assertion returned by .explore. Thus, as for method .explore, if the assertion is not checked then method .recovery returns the assertions that invalidating the ongoing computation. More precisely, the backtracking was already performed during the last computed slice, in accordance to the state-space algorithm. It remains to continue the backtracking from the assertions on the previous slices until the initial slice, i.e., the slice of the initial assertion of the ongoing session. This recovery of the backtracking is performed by the method update $F$ which, as its name indicates, updates the set $F$ of the false assertions. The method also uses the variable $\sigma_{\text {halted }}$ which represent the last assertion computed before the computation of the session was halted. All these methods and a full example are fully available in [26].

\subsubsection{Drawbacks of this naive algorithm}

This naive approach suffers of three main drawbacks. First, each time a session is thrown, it can traverse all the state-space in several super-steps. The number of supersteps would be proportional to the number of states in the Kripke structure. This can happen when the session has been thrown by a formulae which contains modal operators, e.g., a formulae of the form $\mathbf{A A} p$. This is due to the fact that the algorithm works as follow for this formulae: for each state, test if $\mathbf{A} p$ is valid; thus, run each time a LTL session which would implies several super-steps to test $\mathbf{A} p$ (if literal $p$ is valid on all the states of the Kripke structure). This can thus generate too much barriers and induce very poor performance.

Second, the sweep-line strategy used in the previous section cannot be applied: each slice does not correspond to a super-step and thus during backtracking of the answers, the date dumped on disks must be loaded back into the main memory (work of next and previous_slice). This can be very costly. The alternative is to keep everything in the main memory but with a serious risk of swapping.

Third, the balance of the assertions over the processors is done dynamically at each slice of each session: that ensures that two assertions for the same Kripke state are held by the same processor, which avoids duplication of computation. But if two sessions are run in sequence, the first one will balance some assertions and the second session, if some states are shared, must balance the assertions using this first partial scheme of balance which is complicated and largely suboptimal. The alternative to re-balance all the assertions would be too costly. 


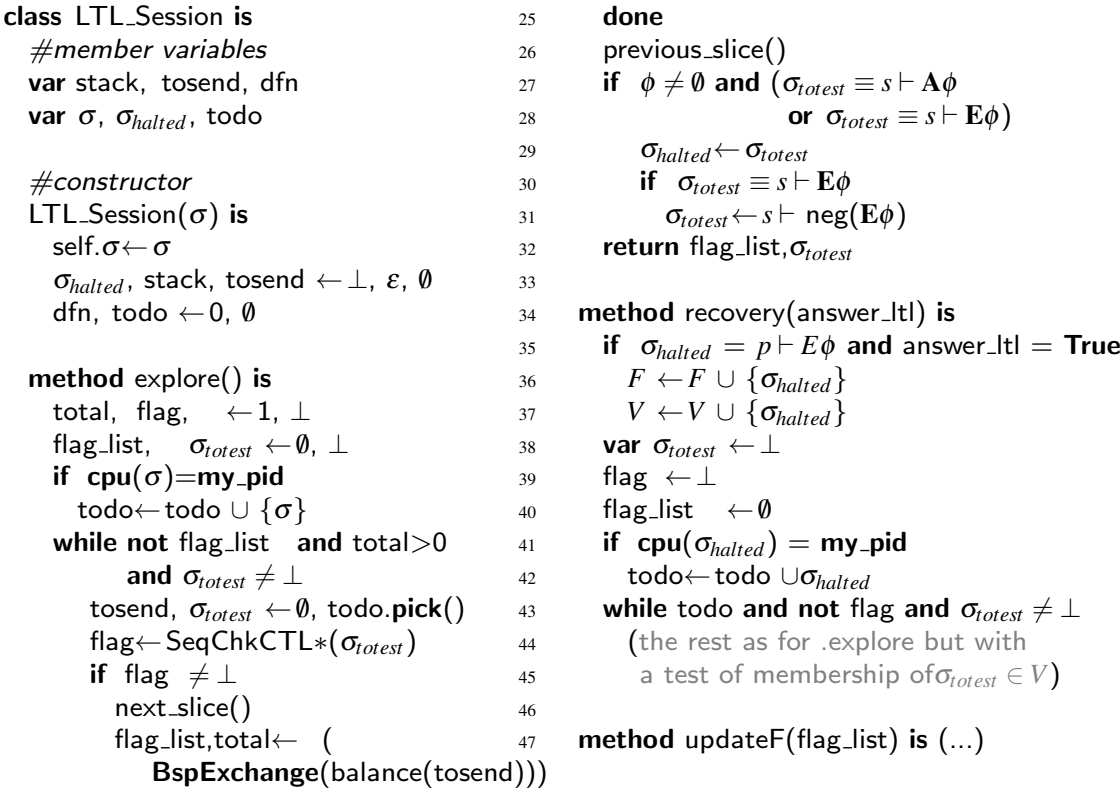

Fig. 17 LTL session for the naive CTL* BSP algorithm.

\subsection{A "purely breadth" BSP algorithm for CTL* checking}

To avoid these problems we will take into account the "nature" of proof-structures that include an explicit decomposition of the logical formulae which can help to choose where a parallel computation is needed or not. The main idea of the algorithm is to consider rules R1 and R2 of Fig. 3 and compute $s \vDash \phi$ together with $s \vdash \mathbf{A}(\Phi)$. This way, we will able to choose which rule ( $R 1$ or $R 2)$ must be applied.

More precisely, in the case of rules $R 1$ and $R 2$ of the decomposition of a LTL formulae, $\phi$ is an atomic proposition, which can thus be sequentially computed. But in the case of $\mathrm{CTL}^{*}, \phi$ can be any formulae. In the naive algorithm, we thus run another LTL session and pause the current computation until a result is provided. The approach proposed for the new algorithm is to compute both $s \vDash \phi$ and $s \vdash \mathbf{A}(\Phi)$, which provides the information to decide whether $R 1$ or $R 2$ must be applied. As previously, the computation of $s \vDash \phi$ can be performed by a kind of LTL session while the computation of $s \vdash \mathbf{A}(\Phi)$ can be performed following the execution of the SCC computation. In a sense, we anticipate the result of $s \vDash \phi$ by computing the validity of the assertion $s \vdash \mathbf{A}(\Phi)$.

We see three main advantages. First, as we can compute both $s \vDash \phi$ and $s \vdash \mathbf{A}(\Phi)$ in parallel, we can aggregate the super-steps of the both computations and thus reduce the overall number of super-steps to the maximal number of slices of the model (slice progression). Second, we also aggregate the computations and the communications without loosing their balance: we have in the same place all the assertions of each slice, which allows a better balancing than the use of the partial balances in the naive algorithm. Third, the computation of the validity of $s \vdash \mathbf{A}(\Phi)$ can be used later in 
different LTL sessions. On the other hand, the pre-computation of $s \vdash \mathbf{A}(\Phi)$ may generate unnecessary work. If we assume a sufficient number of processors, this is not a problem concerning scalability, and the exploration is performed in a breadth fashion that brings a higher degree of parallelism.

\subsubsection{Main loop}

Fig. 18 gives the main loop of the algorithm. The computation is performed until the answer of the initial assertion $\sigma_{0}$ is found, which is recorded in variable finish. The computation works as follows and can be divided into three phases. First (lines 11-18), the current exploration of received assertions (processed one by one in lines 13-17) is performed. Secondly (lines 22-24), the propagation of the backtracks of the answers (not equal to $\perp$ ) found especially on other machines is performed. Note that in the first stage some backtracks of answers can also be performed but they are local and done during the ongoing exploration. Between these two phases, an exchange between the machines is performed (line 20). Finally (line 25), dump from the main memory all the assertions that are no more used for the computation due to slice progression (sweep-line method described latter).

We have thus the overall stack (initially empty) due to our derecursification of the Tarjan algorithm and to the recursive decomposition of the $\mathrm{CTL}^{*}$ formulae. $\mathrm{dfn}$ is the "deep first number" that can be intuitively shared by all SCC decompositions. For the management of the sending assertions, we use two distinct sets of messages. The first one (snd_todo) is to store the assertions which are used to continue the exploration of the distributed proof-structure; The second one (snd_back) is for backtracking answers (for the case of rules R1/R2 expecting the answer about a $s \vDash \phi$ ). This way, at the beginning of a super-step, we first read answers regarding paused sessions (stored in a stack) which could then continue their SCC computations. Then, the algorithm explores the sub-parts of the proof-structures for the received assertions. All these works are done until the initial assertion (of the first session) gets its answer. In the case of a flaw, we rebuild the trace as for LTL checking. This requires a minor change in the global exchange function which also sends answers and globally compute if one processor has finally reached an answer for $\sigma_{0}$.

We thus also modify the function subgoals (see Fig. 18) to take into account the management of the sends, like in our algorithm for LTL checking. Also, we add arcs between the assertions, via the field .pred for each assertion to know its parents, which implicitly gives the graph of the proof-structure. We will use them to backtrack the answers. The function call_ctlstar is modified consequently to manage field .pred. 


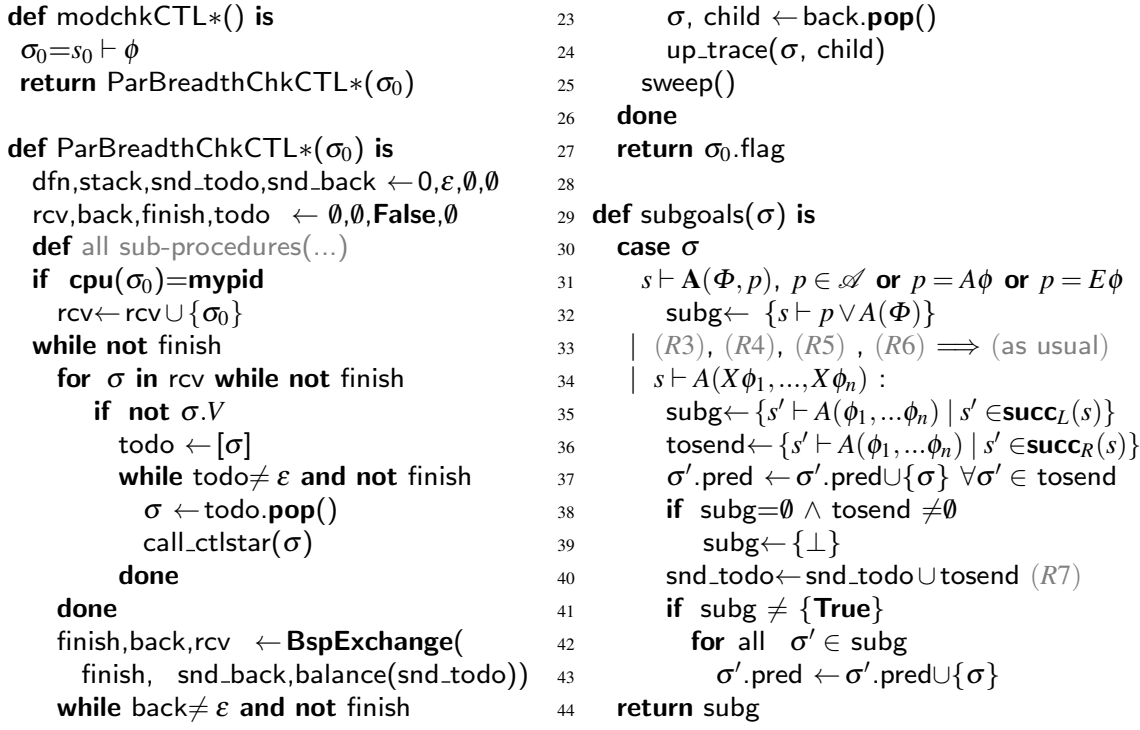

Fig. 18 Main procedure of the breadth $\mathrm{CTL}^{*}$ model-checking algorithm.

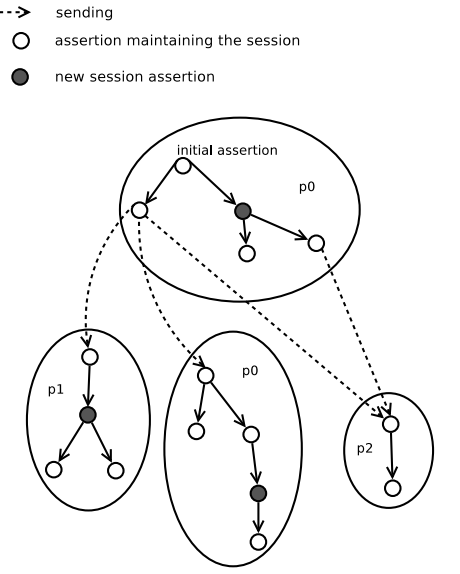

Fig. 19 Breath LTL session generation.
The difficulty in this algorithm is to correctly manage the answers. Indeed, we do not know the answer to an assertion when we compute the validity of $s \vDash \phi$ or when it has been send to another processor. Thus, we need to modify backtracking when an answer is unknown by considering a third possibility of answer: $\perp$, the case when we cannot conclude. This way, the LTL session is paused until an actual Boolean answer is computed, mainly in the next slice (and thus in the next super-step). This is illustrated in Fig. 19 where we have classes of assertions among which some need to start another LTL session (in grey). But in the same classes, we also need to continue the SCC decomposition of the proof-structure in order to keep our slice progression. The development of a new LTL session means that we initiate the generation of a new proof-structure (LTL session) for the checking of an assertion (rules R1/R2). We thus start the new session together with the other sessions whose exploration is in progress, which increases the parallelism.

\subsubsection{Iterative $C T L^{*}$ decomposition}

The backtracking between the LTL sessions and $\mathrm{CTL}^{*}$ is performed using two new fields: .parentCTL* and .parentLTL. Each sent assertion has its fields . parentLTL and . parentCTL* set to $\perp$ since these assertions are not called by their parents, in the 
sens that their parents do not put them onto the stack todo of assertions awaiting for exploration. Note also that procedure call_ctlstar can call procedure call_ttl (line 19) of Fig. 20 which corresponds to starting another LTL session.

We modify the functions call_ctlstar and up_ctlstar accordingly by adding an additional field for each disjunctive and conjunctive assertion: .wait — see Fig. 20. Initially .wait is a set containing the two children of the assertion, like the field .children. If the children of a conjunctive or disjunctive assertion return an answer equal to $\perp$, i.e., each one has an unknown answer, then the child assertion will be removed from the field .children but retained in the field .wait so we know that this assertion has not its answer yet. This trick provides us the answer (possibly $\perp$ ) for the parent assertion.

Take for example the assertion $\sigma \vdash \phi_{1} \vee \phi_{2}$ which has for children $\sigma_{1} \vdash \phi_{1}$ and $\sigma_{2} \vdash \phi_{1}$. Initially, $\sigma$.children $=\sigma$.wait $=\left\{\sigma_{1}, \sigma_{2}\right\}$. Assume that $\sigma$ first calls $\sigma_{1}$, then $\sigma_{1}$ is removed from field $\sigma$.children but is kept in $\sigma$.wait. Field $\sigma$.wait will contain the children assertions for which the answer is not yet known. After some computation, the answer for $\sigma_{1}$ is returned, say $\perp$. Therefore we cannot conclude about $\sigma$. Assume now that $\sigma$ now calls $\sigma_{2} . \sigma_{1}$ is thus removed from field $\sigma$.children. After some computation, the answer for $\sigma_{2}$ is returned, say True. $\sigma_{2}$ is thus removed from field .wait, because its answer is now known. But the field .wait of $\sigma$, containing $\sigma_{1}$ ensures that we can do not conclude, we first have to wait for the answer about $\sigma_{1}$. The procedures work as follows:

- call_ctlstar decomposes the assertions, builds the graph of calls, adds the children into field .wait and finally calls loop_ctlstar (lines 16 and 24 to continue to compute over those assertions) or ret_ctlstar (we have an answer about the assertion) if it is an atomic proposition (line 8);

- loop_ctlstar processes the children by putting them (lines 3-5) in the set of assertions to process (todo), or finishes the computation by a call (line 7) to ret_ctlstar if all the children have been processed;

- ret_ctlstar returns an answer to the appropriate parent if there is one, otherwise,the answer is backtracked using ret_trace (line 7) to all the assertions that expect it (even on other machines by putting the answer in snd_back);

- up_ctlstar computes the answer of an assertion with respect to the answer for its children, possibly concluding even if there are still answers awaited in field wait. For instance, for logical operator $\wedge$, if field flag of one of the children is False (line 11) then the assertion is invalid regardless of other answers that could come later, and so, we can backtrack this new answer by a call to ret_ctlstar (line 14). However, if the answer for a child is True, we have to wait for other answers, until .wait is empty which means that all the children answers have been True.

Note that for procedure call_ctlstar and up_ctlstar, the answer for quantifier $\mathbf{E}$ is the opposite as for $\mathbf{A}$ (we use function neg). The iterative procedures call_ltl, loop_lt|, ret_Itl and up_Itl for SCC decomposition (LTL sessions) and up_trace, ret_trace (for backtracking) are also modified accordingly to take into account the new fields and are fully described and available in [26]. 

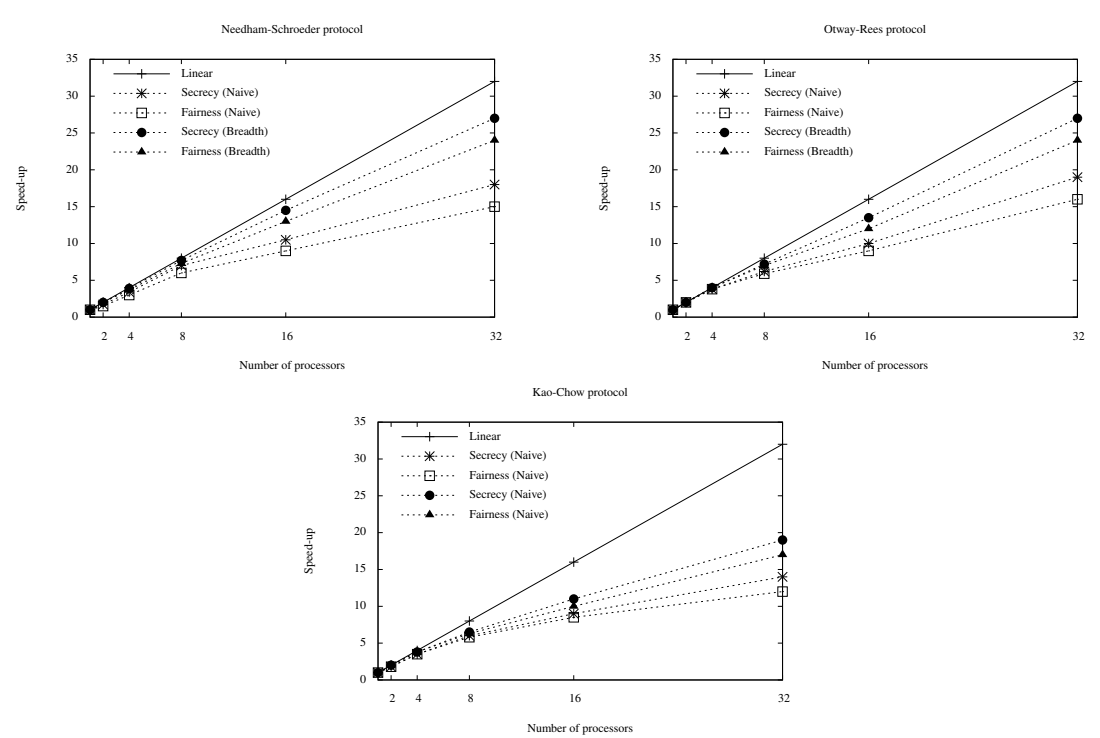

Fig. 21 Speedup results for three of the protocols.
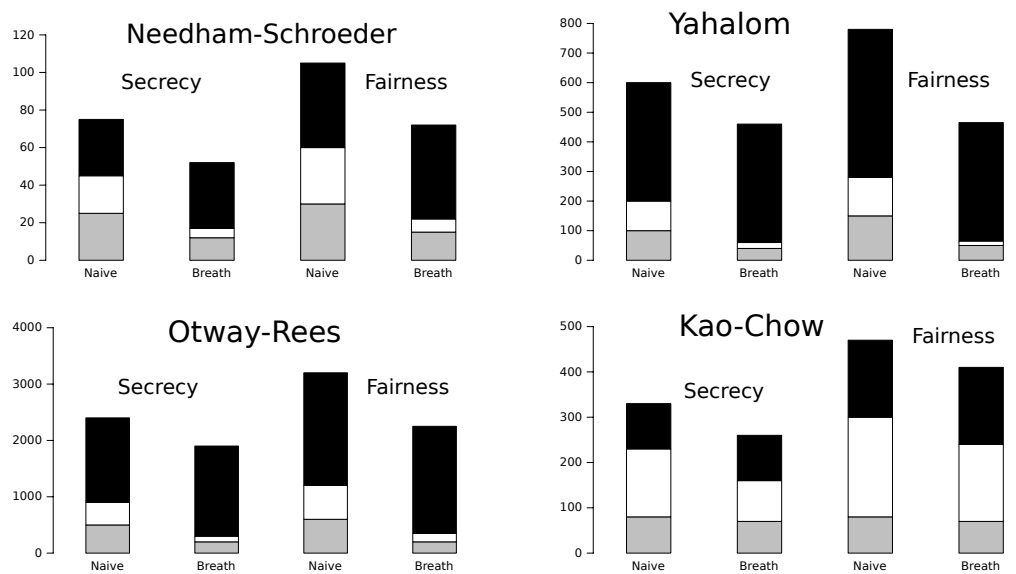

Fig. 22 Timing of the two algorithms ("Naive CTL" " and "Pure Breadth") with respect to formulas Secrecy and Fairness, for the four studied protocols. Times are given in seconds and decomposed as computation time in black, communication time in grey and waiting time in white.

\subsection{Experiments}

In order to evaluate our two algorithms in PYTHON/SNAKES, we have tested two formulas: the first one is the LTL formula [1] for secrecy, whereas the second one is the CTL formula for fairness [29] (as presented above). As previously, the formulas globally hold so that the whole proof-structure is computed.

In Fig. 21, we give the speedup of the two latter algorithms ("Naive CTL" and "Pure Breadth") for three different protocols and for the two formula (as previously, 
results for Yahalom cannot be computed with low number of processors so we have not speedup to show). As we could expect, the naive algorithm scales less for both formula. Note that for Kao-Chow, both algorithms do not scale well. This is mainly due to a lack of possible attacks which implies less classes of states: executions are almost not branching and so the protocol provides very few intrinsic parallelism.

Fig. 22 shows the execution times for our two formulas for each protocol, using 32 processors. In the figure, the total execution time is split into three parts: the computation time (black) that essentially corresponds to the computation of successful SCC of the proof-structures on each processor; the global and thus collective communication time (grey) that corresponds to assertions exchange; the waiting time, i.e., latencies (white) that occur when processors finish their computation early and are forced to wait for the others before to enter the communication phase of each superstep. We can see on these graphs that the overall performance of our "Breath" algorithm is always good compared to the naive one. As expected, the "Breath" algorithm reduce both latencies due to less super-steps and a better balance of communications - since they are more en masse. Fairness needs more computation since it is a more complicated formulae: the bigger the formulas and the model, the better is "Breath" algorithm performs.

\section{Related work}

6.1 Tools and methods for security protocols

Gavin Lowe has discovered the now well-known attack on the Needham-Schroeder public-key protocol using the model-checker FDR [36]. In spite of this, over the last two decades, a wide variety of security protocol analysis tools have been developed that are able to detect attacks on protocols or, in some cases, establish their correctness. We distinguish three classes: tools that attempt verification (proving a protocol correct), those that attempt falsification (finding flaws, i.e., counterexamples), and hybrids that attempt to provide both proofs and counterexamples. In the first category, we find the use of theorem provers [40] and dedicated tools such as PROVERIF [9] or SCYTHER [18], etc., falsification is the domain of model-checking $[6,1]$ — such as the lazy intruder of AVISPA [2].

Paper [19] presents different cases study of verifying security protocols with various standard tools. To summarise, there is currently no tool that provides all the expected requirements.

\subsubsection{Theorem proving for security protocols}

To the best of our knowledge, the first work using theorem proving for verifying security protocols is [40]. And now different researches have been conducted in this way. Using a theorem prover, one formalises the system (the agents running the protocol along with the attacker) as a set of possible communication traces. Afterwards, one can state and prove theorems expressing that the system has certain desirable 
properties, The proofs are usually carried out under strong restrictions, e.g., that all variables are strictly typed and that all keys are atomic.

The main drawback of this approach is that verification is quite time consuming and requires considerable expertise [3]. Moreover, theorem provers provide poor support for error detection when the protocols are flawed, even with the work on integrating automatic methods in theorem provers for security protocols as in [12].

\subsubsection{Dedicated tools}

The first class of tools that focus on verification typically rely on encoding protocols as Horn clauses and applying resolution - without termination guarantee. The most known tool is certainly PROVERIF [9]. The system can handle an unbounded number of sessions of the protocol but performs some approximations - e.g., on random numbers. As a consequence, when the system claims that the protocol preserves the secrecy of a value, this is correct. This tool is thus needed when no flaw has been found in the protocol (with model-checking) and one wants to have a test for an unbounded number of sessions.

Most of dedicated tools limit possible kinds of attacks or limit in their modelling language how agents can be manipulated in ad-hoc protocols. The three main drawbacks of these tools are thus (1) the restricted language used for modelling the protocols; (2) the lack of building traces in case of a flaw (this is not the case using a model-checking method); (3) the limitation of their verification to simple properties (e.g., fairness is generally not taken into account [32]) and of their models essentially limited to "ping-pong" protocols.

\subsubsection{Model-checking of security protocols}

On the contrary, our approach is based on model-checking [6] that is not tied to any particular application domain. Using $\mathrm{CTL}^{*}$, we can also express many complex properties that some dedicated tool cannot. But that also restrict our approach to finite scenario. There are many paper about model-checking of security protocols and the reader can find a gentle survey in [6]. For example, in [38], the authors have used the MURPHI modelling language and different distributed model-checkers for MURPHI now exist. Even if those programs would clearly outperform our prototype tool (due to the use of PYTHON), the algorithm [43] uses a naive random hash function.

For finite scenarios checking (and enumerative state-space construction), the most well known tool is certainly AVISPA [2] that uses dedicated modelling language and algorithms. In contrast, our approach is based on a general modelling framework (algebras of Petri nets) with explicit state-space construction, that is not tight to any particular application domain. Using PYTHON in our implementation allows us to manipulate any kind of data-structures that could be used by agents in protocols. This is a well-desired feature for complex protocols like P2P security protocols in [13]. We believe that our observations and the subsequent optimisations are general enough to be adapted to the model-checkers dedicated to protocol verification: we worked in a very general setting of LTS, defined by an initial state and a successor 
function. Our only requirements are four simple conditions (P1 to P4) that can be easily fulfilled within most concrete modelling formalisms.

\subsection{Distributed and parallel model-checking}

\subsubsection{Distributed and parallel state-space construction}

The main idea of most known approaches to the distributed memory state-space generation is similar to the "naive" algorithm of [24] which usually introduces too many cross-transitions. More references can be found in [22].

Examples from the literature is the various techniques used in order to avoid sending a state away from the current processor if its 2nd-generation successors are local. This is complemented with a mechanism that prevents from re-sending already sent states. The idea is to compute the missing states when they become necessary for model-checking, which can be faster than sending it. That clearly improves communications but our method achieves similar goals, in a much simpler way, without ignoring any state. Close to our hashing technique, [41] presents a hashing function that ensures that most of the successors are local: the partition function is computed by a round-robin on the successor states. This improves the locality of the computations but can duplicate states. Moreover, it only works well when network communication is substantially slower than computation, which is not the case on modern parallel architectures. We can also find a balancing strategy in [15] where a balance is performed each time the system detects too many states on a node. That is not needed and would imply too much communication in our case.

In [34], a distributed state-space algorithm derived from the SPIN model-checker is implemented using a master/slave paradigm. Several SPIN-specific partition functions are experimented, the most advantageous one being a function that takes into account only a fraction of the state vector, similarly to $\mathrm{cpu}_{\mathscr{R}}$. The algorithm performs well on homogeneous networks of machines, but does not outperform the standard implementation, except for problems that do not fit into the main memory of a single machine. Moreover, no clue is provided about how to choose correctly the fraction of states that has to be considered for hashing, while we have relied on reception locations from $\mathscr{R}$. SPIN has also been used for verifying security protocols [37].

In [39], an user-defined abstract interpretation is used to reduce the size of the state-space, and so it allows to distribute the abstract graph; the concrete graph is then computed in parallel for each part of the distributed abstract graph. In contrast, our distribution method is fully automated and does not require input from the user.

In [10] authors used complex distributed file systems or shared databases to optimise the sending of the states, especially when complex data-structure are used internally in the states - as ours. That can improve our implementation but not the idea of the method. In [21], the authors used heuristics for the sweep-line method with the drawback that these heuristics can fail. In our case of security protocols, no such heuristic is necessary since the structured model gives the progression. 


\subsubsection{Distributed and parallel temporal logic verification}

If model-checking of LTL formula has been the more studied, works for CTL can be found in [11] and in [35] for the $\mu$-calculus (which is more expressive than $\mathrm{CTL}^{*}$ ).

Close to our idea of localising cycles, we can cite [4] which both used partition functions that enable cycles to be local only - as for us. The limits of the method are the cost of their functions as well as the number of SCCs which is not sufficient to scale. [5] presents distributed algorithms for SCC computation. In our work, all SCCs are purely local, which is easier to handle and more efficient.

A kind of tree (hesitant) Büchi automata is used in [30] where parallel SCC computations are performed. The automaton is hesitant is the sense that as for rules R1 and R2, it cannot conclude and thus initiates the two possible computations. That generated what they call "games" (close to our "sessions") and the algorithm has to manage how to store partial results of games. Shared memory computations and heuristics are used here to simplify this management. The algorithm has also expensive management of invalid SCCs, which seems not feasible for a distributed architecture. These algorithms have also been tested to check security protocols in [29].

\section{Conclusion and future work}

Designing security protocols is complex and error prone: various attacks are reported in the literature to protocols thought to be "correct" for many years. There are now many tools that check the security of cryptographic protocols and model-checking is one solution. It is mainly used to find flaws in finite scenario (bounded number of agents) but not to prove the correctness of a protocol. To check if scenario contains flaw or not, we thus propose to resort to explicit distributed model-checking, using an algebra of coloured Petri nets to model the protocol, together with security properties that could be expressed as reachability properties, LTL, or CTL* formulas. Reachability properties lead us to construct the state-space of the model (i.e., the set of its reachable states). LTL and $\mathrm{CTL}^{*}$ involve the construction of the state graph (i.e., the reachable states together with the transitions from states to others) which is combined with the formula under analysis into a so-called proof-structure. In both cases, on-the-fly analysis allows to stop states explorations as soon as a conclusion is drawn.

Using a distributed algorithm is a common solution to benefit from more memories and computations units. But, the critical problem of state-space construction is to determine whether a newly generated state has been explored before. In a serial implementation this question is answered by organizing known states in a specific data-structure, and looking for the new states in that structure. As this is a centralized activity, a parallel or distributed solution must find an alternative approach. The common method is to assign states to processors using a static partition function which is generally a hashing of the states. After a state has been generated, it is sent to its assigned location, where a local search determines whether the state already exists. Applying this method to security protocols fails in two points. First the number of cross-transitions (i.e., transitions between two states assigned to distinct processors) is too high and leads to a too heavy network use. Second, memorizing all of them in 
the main memory is impossible without crashing the whole parallel machine and is not clear when it is possible to put some states in disk and if heuristics (e.g., a caching strategy) would work well for complex protocols.

Our parallel algorithm for the state-space computation (basis of model-checking) of the finite scenario of protocols, use the well-structured nature of the protocols in order to choose which part of the state-space is really needed for the partition function and to empty the data-structures in each super-step of the parallel computation. The state-space is thus distributed in such a way that there is no roll-back in the super-step, which allows to divise the state-space into slices and ensures that there is no cross-transitions during local computations. Our algorithms also entail automated classification of states into classes, and the dynamic mapping of classes to processors. We find that both our methods execute significantly faster than the traditional one and achieve a better network use, memory balance and computation time.

With these properties in mind, we have designed two algorithms for verifying temporal logical formula over finite scenario of protocols, one for LTL checking and another one for $\mathrm{CTL}^{*}$ checking. Both are parallelisation of an existing algorithm based on building proof-structures and computating strongly connected components (SCCs) using a Tarjan like method. The structure of state-space exploration is thus preserved but enriched with the construction of the proof-structure and its on-the-fly analysis. This allows parallel machines to apply automated reasoning techniques, to perform a formal analysis of security protocols. In the case of LTL, we have seen that no cross-transition occurs within a SCC, which is crucial to conclude about formula truth value. In the case of $\mathrm{CTL}^{*}$ however, local conclusions may need to be delayed until a further recursive exploration is completed, which might occur on another processor. Rather than continuing such an exploration on the same processor, which would limit parallelism, we designed a way to organise the computations so that inconclusive nodes in the proof-structure can be kept available until a conclusion is drawn from a recursive exploration, allowing to dump them immediately from the main memory. This more complex bookkeeping appears necessary due to the recursive nature of $\mathrm{CTL}^{*}$ checking that can be regarded as nested LTL analysis.

The fundamental message is that for parallel model-checking, exploiting certain characteristics of the system and structuring the computation is essential. We have demonstrated techniques that proved the feasibility of this approach and demonstrated its potential. Key elements to our success were: (1) an automated classification that reduces cross-transitions and memory use and growth locality of the computations; (2) using global barriers (which is a low-overhead method) to compute a global remapping and thus balancing workload and achieved a good scalability for the statespace generation of security protocols; (3) careful extension of this state-space algorithm to handle the case of LTL first, then CTL*.

Future works will be dedicated to build an efficient implementation from our prototypes. Using it, we would like to run benchmarks in order to compare our approach with existing tools such as AVISPA, which is currently meaningless due to our PYTHON implementations. Using BSP-PYTHON is good for a short developpement cycle of the prototypes but that generates inefficient parallel programs. We would like also to test our algorithms on parallel computers with more processors in order to confirm the scalability observed on 40 processors. More practically: we would like 
to have a tool able to translate HSPSL models [1] (a standard language for describing security protocols) to ABCD ones since HSPSL is mainly used by the community.

Finally, we would like to generalise our present results by extending its application domain to more complex protocols with branching and looping structures, as well as complex data types manipulations as in protocols for secure storage distributed through peer-to-peer communication [13], secured routing protocols [17], etc.

\section{References}

1. Armando, A., Carbone, R., Compagna, L.: Ltl model checking for security protocols. Applied NonClassical Logics 19(4), 403-429 (2009)

2. Armando, A., et al.: The AVISPA tool for the automated validation of Internet security protocols and applications. In: K. Etessami, S.K. Rajamani (eds.) Proceedings of Computer Aided Verification (CAV), LNCS, vol. 3576, pp. 281-285. Springer (2005)

3. Backes, M., Unruh, D.: Limits of constructive security proofs. In: J. Pieprzyk (ed.) Theory and Application of Cryptology and Information Security (ASIACRYPT), LNCS, vol. 5350, pp. 290-307. Springer (2008)

4. Barnat, J., Brim, L., Cëerná, I.: Property driven distribution of nested dfs. In: M. Leuschel, U. UltesNitsche (eds.) Workshop on Verification and Computational Logic (VCL), vol. DSSE-TR-2002-5, pp. 1-10. Dept. of Electronics and Computer Science, University of Southampton (DSSE), UK, Technical Report (2002)

5. Barnat, J., Chaloupka, J., Pol, J.V.D.: Distributed Algorithms for SCC Decomposition. Journal of Logic and Computation 21(1), 23-44 (2011)

6. Basin, D., Cremers, C., Meadows, C.: Model Checking Security Protocols, chap. 24. Springer (2011)

7. Bhat, G., Cleaveland, R., Grumberg, O.: Efficient on-the-fly model checking for ctl*. In: Proceedings of the 10th Annual IEEE Symposium on Logic in Computer Science (LICS), pp. 388-398. IEEE Computer Society (1995)

8. Bisseling, R.H.: Parallel Scientific Computation. A structured approach using BSP and MPI. Oxford University Press (2004)

9. Blanchet, B.: An efficient cryptographic protocol verifier based on Prolog rules. In: IEEE CSFW'01. IEEE Computer Society (2001)

10. Blom, S., Lisser, B., van de Pol, J., Weber, M.: A database approach to distributed state-space generation. J. Log. Comput. 21(1), 45-62 (2011)

11. Boukala, M.C., Petrucci, L.: Distributed model-checking and counterexample search for ctl logic. IJCCBS 3(1/2), 44-59 (2012)

12. Brucker, A.D., Mödersheim, S.: Integrating automated and interactive protocol verification. In: Formal Aspects in Security and Trust (FAST), LNCS, vol. 5983, pp. 248-262. Springer (2009)

13. Chaou, S., Utard, G., Pommereau, F.: Evaluating a peer-to-peer storage system in presence of malicious peers. In: W.W. Smari, J.P. McIntire (eds.) High Performance Computing and Simulation (HPCS), pp. 419-426. IEEE (2011)

14. Christensen, S., Kristensen, L.M., Mailund, T.: A sweep-line method for state space exploration. In: T. Margaria, W. Yi (eds.) Proceedings of Tools and Algorithms for the Construction and Analysis of Systems (TACAS), LNCS, vol. 2031, pp. 450-464. Springer (2001)

15. Ciardo, G., Gluckman, J., Nicol, D.M.: Distributed state space generation of discrete-state stochastic models. INFORMS Journal on Computing 10(1), 82-93 (1998)

16. Comon-Lundh, H., Cortier, V.: How to prove security of communication protocols? a discussion on the soundness of formal models w.r.t. computational ones. In: STACS, pp. 29-44 (2011)

17. Cortier, V., Degrieck, J., Delaune, S.: Analysing routing protocols: Four nodes topologies are sufficient. In: P. Degano, J.D. Guttman (eds.) Principles of Security and Trust (POST), LNCS, vol. 7215, pp. 30-50. Springer (2012)

18. Cremers, C.J.F.: Scyther - semantics and verification of security protocols. Ph.D. thesis, Technische Universiteit Eindhoven (2006)

19. Cremers, J.F., Lafourcade, P., Nadeau, P.: Comparing state spaces in automatic security protocol analysis. In: Formal to Practical Security, LNCS, vol. 5458, pp. 70-94. Springer (2009) 
20. Dolev, D., Yao, A.C.: On the security of public key protocols. IEEE Transactions on Information Theory 29(2), 198-208 (1983)

21. Evangelista, S., Kristensen, L.M.: Hybrid on-the-fly ltl model checking with the sweep-line method. In: S. Haddad, L. Pomello (eds.) Application and Theory of Petri Nets, LNCS, vol. 7347, pp. 248-267. Springer (2012)

22. Ezekiel, J., Lüttgen, G.: Measuring and evaluating parallel state-space exploration algorithms. Electr. Notes Theor. Comput. Sci. 198(1), 47-61 (2008)

23. Fokkink, W., Dashti, M.T., Wijs, A.: Partial order reduction for branching security protocols. In: L. Gomes, V. Khomenko, J.M. Fernandes (eds.) Conference on Application of Concurrency to System Design (ACSD), pp. 191-200. IEEE Computer Society (2010)

24. Garavel, H., Mateescu, R., Smarandache, I.M.: Parallel state space construction for model-checking. In: M.B. Dwyer (ed.) Proceedings of SPIN, LNCS, vol. 2057, pp. 217-234. Springer (2001)

25. Goranko, V., Kyrilov, A., Shkatov, D.: Tableau tool for testing satisfiability in ltl: Implementation and experimental analysis. Electr. Notes Theor. Comput. Sci. 262, 113-125 (2010)

26. Guedj, M.: Bsp algorithms for $1 t 1$ \& ctl* model checking of security protocols. Ph.D. thesis, University of Paris-Est (2012)

27. Hinsen, K.: Parallel scripting with Python. Computing in Science \& Engineering 9(6) (2007)

28. Holzmann, G., Peled, D., Yannakakis, M.: On nested depth first search (extended abstract). In: The Spin Verification System, pp. 23-32. American Mathematical Society (1996)

29. Inggs, C., Barringer, H., Nenadic, A., Zhang, N.: Model checking a security protocol. In: Southern African Telecommunications Network and Applications Conference (SATNAC) (2004)

30. Inggs, C.P., Barringer, $\mathrm{H} .: \mathrm{Ctl}^{*}$ model checking on a shared-memory architecture. Formal Methods in System Design 29(2), 135-155 (2006)

31. Iosup, A., Sonmez, O., Anoep, S., Epema, D.: The performance of bags-of-tasks in large-scale distributed systems. In: Symposium on High performance distributed computing (HPDC), pp. 97-108. ACM (2008)

32. Kremer, S., Markowitch, O., Zhou, J.: An intensive survey of fair non-repudiation protocols. Computer Communications 25(17), 1606-1621 (2002)

33. Kumar, R., Mercer, E.G.: Load balancing parallel explicit state model checking. In: ENTCS, vol. 128, pp. 19-34. Elsevier (2005)

34. Lerda, F., Sista, R.: Distributed-memory model checking with SPIN. In: D. Dams, R. Gerth, S. Leue, M. Massink (eds.) Proceedings of SPIN, no. 1680 in LNCS, pp. 22-39. Springer-Verlag (1999)

35. Leucker, M., Somla, R., Weber, M.: Parallel model checking for ltl, ctl*, and 1. Electr. Notes Theor. Comput. Sci. pp. 1-1 (2003)

36. Lowe, G.: Breaking and fixing the needham-schroeder public-key protocol using fdr. In: T. Margaria, B. Steffen (eds.) Tools and Algorithms for Construction and Analysis of Systems (TACAS), LNCS, vol. 1055, pp. 147-166. Springer (1996)

37. Maggi, P., Sisto, R.: Using spin to verify security properties of cryptographic protocols. In: D. Bosnacki, S. Leue (eds.) Model Checking of Software (SPIN), LNCS, vol. 2318, pp. 187-204 Springer (2002)

38. Mitchell, J.C., Mitchell, M., Stern, U.: Automated analysis of cryptographic protocols using murphi. In: IEEE Symposium on Security and Privacy, pp. 141-151. IEEE Computer Society (1997)

39. Orzan, S., van de Pol, J., Espada, M.: A state space distributed policy based on abstract interpretation. In: ENTCS, vol. 128, pp. 35-45. Elsevier (2005)

40. Paulson, L.C.: The inductive approach to verifying cryptographic protocols. Journal of Computer Security 6(1-2), 85-128 (1998)

41. Petcu, D.: Parallel explicit state reachability analysis and state space construction. In: Proceedings of ISPDC, pp. 207-214. IEEE Computer Society (2003)

42. Pommereau, F.: Algebras of coloured Petri nets. Lambert Academic Publisher (2010). ISBN 978-38433-6113-2

43. Stern, U., Dill, D.L.: Parallelizing the murj verifier. Formal Methods in System Design 18(2), 117-129 (2001) 